\title{
Toward an Empirical Theory of Pulsar Emission. IX. On the Peculiar Properties and Geometric Regularity of Lyne \& Manchester's "Partial Cone" Pulsars
}

\author{
Dipanjan Mitra ${ }^{1}$ \\ National Astronomy and Ionosphere Center, Arecibo Observatory, HC3 Box 53995, PR 00612 \\ and \\ Joanna M. Rankin ${ }^{2}$ \\ Sterrenkundig Instituut "Anton Pannekoek," University of Amsterdam, Science Park 904, 1098 XH \\ Amsterdam, Netherlands \\ Released 2004 Xxxxx XX
}

\begin{abstract}
Lyne \& Manchester (1988) identified a group of some 50 pulsars they called "partial cones" which they found difficult to classify and interpret. They were notable for their asymmetric average profiles and asymmetric polarization position-angle (PPA) traverses, wherein the steepest gradient (SG) point fell toward one edge of the total intensity profile. Over the last two decades, this population of pulsars has raised cautions regarding the core/cone model of the radio pulsaremission beam which implies a high degree of order, symmetry and geometric regularity.

In this paper we reinvestigate this population "partial cone" pulsars on the basis of new single pulse polarimetric observations of 39 of them, observed with the Giant Meterwave Radio Telescope in India and the Arecibo Observatory in Puerto Rico. These highly sensitive observations help us to establish that most of these "partial cones" exhibit a core/cone structure just as did the "normal" pulsars studied in the earlier papers of this series. In short, we find that many of these "partial cones" are partial in the sense that the emission above different areas of their polar caps can be (highly) asymmetric. However, when studied closely we find that their emission geometries are overall identical to core/double cone structure encountered earlier - that is, with specific conal dimensions scaling as the polar cap size.

Further, the "partial cone" population includes a number of stars with conal single profiles that are asymmetric at meter wavelengths for unknown reasons (e.g., like those of B0809+74 or B0943+10). We find that aberration-retardation appears to play a role in distorting the core/cone emission-beam structure in rapidly rotating pulsars. We also find several additional examples of highly polarized pre- and postcursor features that do not appear to be generated at low altitude but rather at high altitude, far from the usual polar fluxtube emission sites of the core and conal radiation.
\end{abstract}

Subject headings: miscellaneous - methods:MHD — plasmas — data analysis — pulsars: general, individual — radiation mechanism: nonthermal - polarization

\footnotetext{
${ }^{1}$ National Centre for Radio Astrophysics, Ganeshkhind, Pune 411007 India: dmitra@ncra.tifr.res.in

${ }^{2}$ Physics Department, 82 University Place, Uni-
} 


\section{Introduction}

Early investigators were impressed by the symmetrical emission profiles of many pulsars (e.g., Backer 1976) and that these, together with their antisymmetic polarization-angle (hereafter PPA) traverses (Radhakrishnan \& Cooke 1969), appeared to reflect their polar cap emission geometry directly. Indeed, the major purpose and overall theme of this "Empirical Theory" series has been that of demonstrating the geometric orderliness of most pulsar emission. Species of profiles were defined in Paper I (e.g., Rankin 1983a; see References for further series numbers). The geometric regularities of core components in relation to the polar cap was introduced in Paper IV, and Paper VI then presented a full quantitative analysis of pulsar emission geometry using the core-double cone model of some 200 stars 1

Certain "difficult" pulsars raised the possibility, even from Paper I, that the polar cap emission from some pulsars might be very asymmetric. Only a few possible examples of one-sided "triple" profiles were mentioned, however, given the difficulty of demonstrating that "double" profiles might sometimes present only a single component. The term "partial cones" was then introduced by Lyne \& Manchester (hereafter L\&M) in their 1988 radio-pulsar beamform study to describe a group of profiles that were not easily classified as falling into one of their cone- or coredominated categories. They confirmed that the majority of their 200 or so pulsars showed a highly ordered, roughly symmetric, quantitatively consistent beam geometry. By contrast, their largish residuum of pulsars with unclassifiable, asymmetric profiles were dubbed "partial cones", because a number (e.g., B0540+23) had asymmetric profiles reminiscent of one side of a classic conal double profile (e.g., B0525+21). This aberrant group of pulsars raised strong cautions - indeed, if some

\footnotetext{
${ }^{1}$ Commonalities in terms of spectral behavior and modulation were studied in Papers II and III. Three other numbers (Papers V, VII and VIII) have respectively discussed circular polarization, radio-to-frequency mapping, and edge depolarization. The results of Paper VI were sufficiently surprising that several groups carried out critical studies or independent analyses (Bhattacharya \& van den Heuvel 1991, Gil et al 1993; Kramer et al 1994; Mitra \& Deshpande 1999), and the core/double cone model of pulsar emission profiles was fully vetted.
}

$20-30 \%$ of all profiles cannot be classified in terms of cores and cones, is this model not itself suspect? Given these patently inscrutable profiles, often with puzzling asymmetries, they left open the possibility that a "patchy" pattern of components resulted from "hot spots" on the polar cap.

We thus reemphasize that L\&M's work and ours provide highly compatible geometrical results for a majority of pulsars in our largely common population, so the differing interpretations of the two analyses turn importantly on L\&M's group of "partial cone" pulsars.

No further systematic study of L\&M's "partial cones" has been carried out over the last two decades, so this group of some 60 pulsars remains in many workers minds as strong evidence for unsystematic pulsar beaming and perhaps polar-cap "hot spots". L\&M's study was based solely on average profiles, most all of them at meter wavelengths, and the general weakishness of this population also limited the quality of their profiles. Now, however, not only are much more sensitive observations often possible - and at both higher and lower frequencies - but pulse-sequence (hereafter PS) polarimetry has been carried out for a large fraction of these "partial cones".

Surely we concur that many of L\&M's "partial cone" pulsars present particular difficulties of interpretation. We now know with certainty that some pulsars do illuminate their polar caps very asymmetrically or episodically (Rankin et al 2006a) - producing lopsided or distorted profilesbut when investigated in detail these stars also exhibit orderly profile dimensions in relation to the polar cap.

A further set of pulsars with conal single profiles, we now know from detailed studies, very often exhibit highly asymmetric profiles (e.g., B0943+10; Deshpande \& Rankin 2001) despite strong evidence that their emission cones are produced by subbeam carousels rotating through our sightline. Aberration/retardation (hereafter A/R) effects have been identified in a number of slower pulsars (e.g., Blaskiewicz et al 1991), and clearly may have strong effects in faster pulsars. Also, recent researches have revealed highly polarized profile features - e.g., the "precursors" in pulsars B0943+10 and B1822-09 (see Backus et al 2010) and even the entire profiles of particular stars (e.g., B0656+14, Weltevrede et al 2006a) that exhibit 
such dissonant properties that we are forced to question whether some new non-core/cone emission process is entailed!

Generally the average profile of a radio pulsar has a characteristic steep outer edge, which apparently reflects the emitting region along the boundary of the "open" magnetosphere (or polar flux tube) adjacent to the closed field region. For a large number of pulsars the polarizationposition angle (PPA) across the pulse profile is seen to execute a smooth 'S-shaped' curve, which according to the rotating-vector model (RVM) proposed by Radhakrishnan \& Cooke (1969) is taken as evidence for emission arising within the polar flux tube and centered around the magnetic axis. Within the RVM the steepest-gradient (hereafter SG) point (or the point of inflection) of the 'S-shaped' curve is interpreted as the plane containing the magnetic dipole axis, and is often located towards the center of the profile.

Most profiles, however, tend to be asymmetric with the central core component of triple or fivecomponent forms seen to lag the centers of their conal-component pairs. Studies by Malov \& Suleymanova (1998), Gangadhara \& Gupta (2001), Gupta \& Gangadhara (2003), Mitra \& Li (1999), and Dyks et al (2004) demonstrate that aberration/retardation (hereafter $\mathrm{A} / \mathrm{R}$ ) effects arising due to emission from a finite height within the pulsar magnetosphere can give rise to the observed profile asymmetries. Once this A/R effect is properly taken into account, the emission can be understood as nested conal emission.

Partial cones were identified by L\&M as pulsars with profiles having one steeply rising edge and another slowly falling edge. Or, as stars where the steepest gradient point of the PPA traverse is located towards one edge of the profile. Identification of partial cones thus requires unambiguous determination of the SG point of the PPA swing with respect to its total intensity profile. It is often difficult to discern the character of the PPA traverse using only average-profile polarimetry, as did L\&M. This is particularly so due to the presence of the "orthogonal" polarization modes (hereafter OPMs), which indeed are not always orthogonal (e.g., Ramachandran et al 2004). Departures from modal orthogonality tend to produce complex average PPA behaviors, because their relative power often varies strongly with pulse longitude, and these can in turn lead to serious misinterpretations of a pulsar's PPA traverse. Hence, polarimetry of individual pulses is necessary to distinguish the OPMs and correctly assess the geometrical bases of the PPA swings (e.g., Gil \& Lyne 1995).

L\&M suggested that partial cones are perhaps pulsars where only part of the polar cap is illuminated. "Partial cones" surely do present difficulties for the core-cone beam model. However, the ubiquity of subpulse modulation (e.g., Weltevrede et al 2006b, 2007), implying that cones are generally produced by rotating subbeam "carousels", also raises strong contradictions in any appeal to "hot spots" 2

Below we will argue that the emission from a number of "partial cone" pulsars is indeed partial in the sense that their emission is very asymmetric with respect to the longitude of the magnetic axis. However, we also find that the "partial cone" pulsars are completely regular in terms of their overall core/double cone emission geometry when these asymmetries are accounted for. Or, said differently, we do not yet understand why pulsar radiation is in some cases so beautifully symmetric and in other pulsars so utterly asymmetric about the magnetic axis. However, in most cases it is possible to discern some weak or occasional emission even from the dimmer parts of the polar cap - and the core/double cone geometry of this emission is identical to that of "normal" pulsars.

We then proceed as follows: $\S I I$ describes our GMRT and Arecibo observations, and in §III we discuss our analyses of those pulsars exhibiting "flared" or episodic emission. §IV presents the large subset of "partial cone" pulsars with narrow conal profiles. In $\S \mathrm{V}$ we introduce new analyses of pulsars with clear signatures of $A / R$ in their emission, and in §VI we discuss the several stars with apparently aberrant polarizationcomponents with nearly complete linear and flat PPA traverses. §VII then presents our overall geometrical analyses, and $\S$ VIII gives a summary and discussion of our results. The Appendix then discusses the properties of L\&M's "partial cone" population individually.

\footnotetext{
${ }^{2}$ Even for the recent Karasterigiou \& Johnston (2007) hybrid model, where the conal emission ring is illuminated in patches.
} 

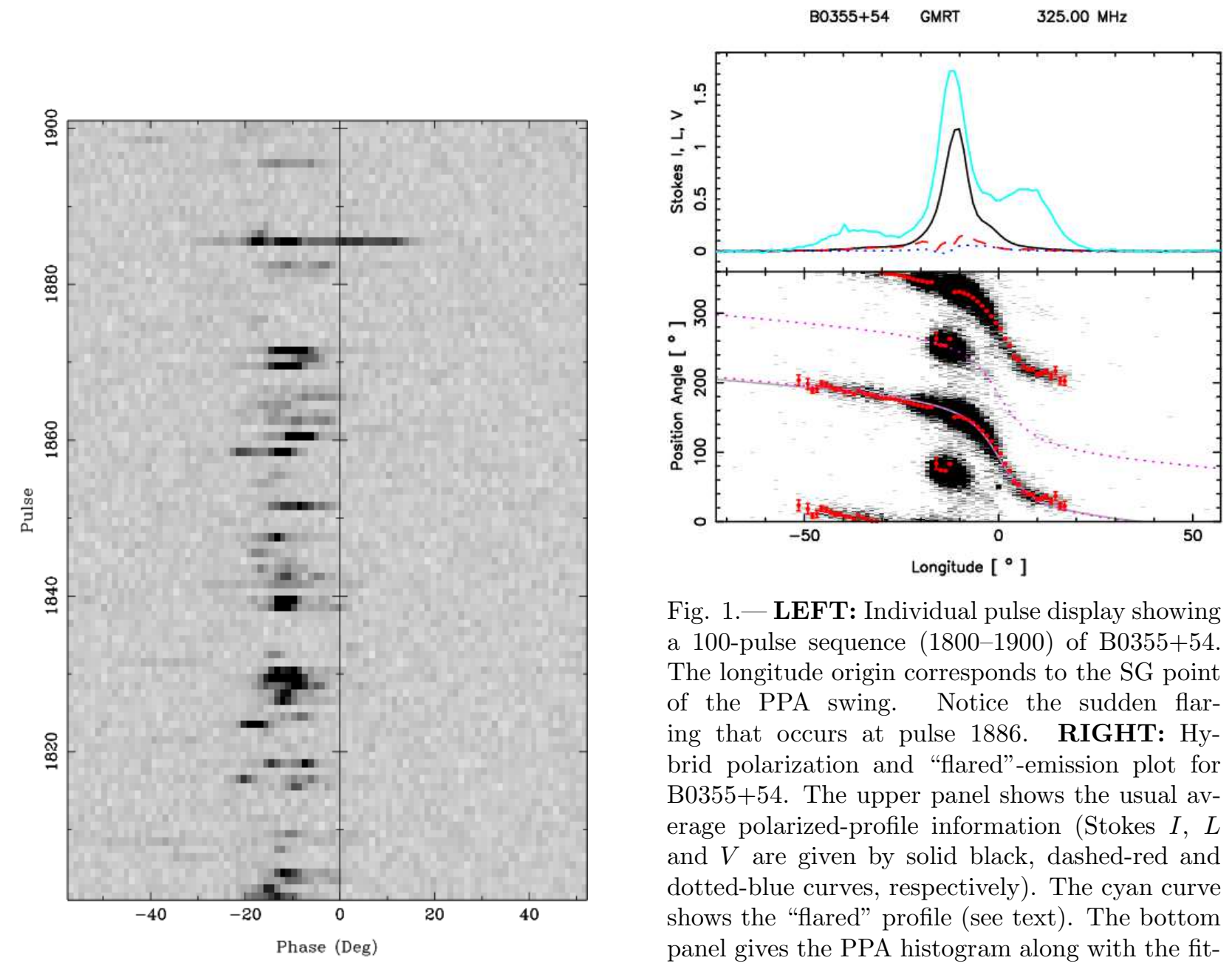

Fig. 1.- LEFT: Individual pulse display showing a 100-pulse sequence (1800-1900) of B0355+54. The longitude origin corresponds to the SG point of the PPA swing. Notice the sudden flaring that occurs at pulse 1886. RIGHT: Hybrid polarization and "flared"-emission plot for B0355+54. The upper panel shows the usual average polarized-profile information (Stokes $I, L$ and $V$ are given by solid black, dashed-red and dotted-blue curves, respectively). The cyan curve shows the "flared" profile (see text). The bottom panel gives the PPA histogram along with the fitted RVM curves; one (dotted magenta, shown for both polarization modes) reflects the geometrical models in Table A3 whereas the other (solid grey) corresponds to the fitting results in Table $\mathrm{A5}$. 


\section{Observations and Data Analysis}

Our observations encompass of 39 of the 50 pulsars identified as "partial-cone" or likely "partialcone" objects by L\&M (their tables 4 \& 5). We have observed these pulsars using the Giant Meterwave Radio Telescope (GMRT) at $325 \mathrm{MHz}$ ( $\mathrm{P}$ band) and the Arecibo (AO) instrument at $\mathrm{P}$ and/or L (1100-1700 MHz) band in full polarization.

The GMRT (Swarup et al 1991) is an array of 30 45-m antennas, spread over a $25-\mathrm{km}$ region $80 \mathrm{~km}$ north of Pune, India. It is primarily an aperturesynthesis interferometer but can also be used in a phased-array configuration. The GMRT operates at multiple frequencies $(150,235,325,610$ and $1000-1450 \mathrm{MHz}$ ) and has a maximum bandwidth of $32 \mathrm{MHz}$, split into upper and lower sidebands of $16 \mathrm{MHz}$ each. At $325 \mathrm{MHz}$, which is the frequency of interest here, the feeds are linearly polarized and converted to circulars using a hybrid. Our observations were carried out on 14 February 2006 and 26 October 2007 using the phased-array mode (Sirothia 2000; Gupta et al 2000), in which the voltage signals of the upper sidebands from each antenna were first added coherently and then fed to the pulsar receiver. The pulsar backends computed the auto- and cross-polarized power between the two circularly polarized signals, and these were finally recorded with a sampling time of 0.512 msec. A suitable calibration procedure as described in Mitra et al (2005) was applied to the observations to recover the calibrated Stokes parameters $I, Q, U$ and $V$. The AO observations were carried out at both 327 and $1400 \mathrm{MHz}$ in a manner very like that reported in Rankin et al (2006a).

The calibrated Stokes parameters were used to compute the total linear polarization $L(=$ $\left.\sqrt{U^{2}+Q^{2}}\right)$ and the PPA $\chi\left(=0.5 \tan ^{-1}(U / Q)\right)$ of the several pulse sequences (hereafter PSs). Table A1 gives the various observational parameters for "partial-cone" pulsars. Table A2 then reviews some of the properties of these "partial cone" pulsars.

In a number of cases we have fitted the RVM to the PPA $\chi$ traverses using the Everett \& Weisberg
(2001) convention as follows:

$\chi=\tan ^{-1}\left(\frac{\sin \alpha \sin \left(\varphi-\varphi_{\circ}\right)}{\sin \xi \cos \alpha+\cos \xi \sin \alpha \cos \left(\varphi-\varphi_{\circ}\right)}\right)+\chi_{\circ}$

where $\alpha$ is the magnetic latitude, $\beta$ is the sightline impact angle, $\xi=\alpha+\beta$ is the sightline-circle radius, and $\chi_{\circ}$ and $\varphi_{\circ}$ are the PPA and longitude offsets. The fits determined four parameters, $\alpha$ and $\beta$ as well as the two offsets above in terms of the overall PPA as a function of longitude. As is usual and well known, the $\alpha$ and $\beta$ values were usually so poorly determined (large errors) and highly correlated (typically 98\%) that they were meaningless. We however use the fitting process to determine the errors in the fitted parameters by evaluating the amount of change required for a particular parameter (holding the other parameters fixed) such that the minimum chi-square value increases by unity (see von Hoensbroech \& Xilouris 1997, Everett \& Weisberg 2001, Mitra $\&$ Li 2004). This way the PPA sweep rate $R$ $\left[=|\Delta \chi / \Delta \phi|_{0}=\sin (\alpha) / \sin (\beta)\right]$ and longitude offset "steepest gradient" (hereafter SG) point $\varphi_{\circ}$ were often well determined. Table A5 gives these latter RVM fitted/computed values and their respective errors. From this fitting exercise, the quantity $R$ is obtained for the geometric analyses that are summarized in Table $\mathrm{A3}$ and discussed in $\S$ VII. It is to be noted that the errors in $R$ quoted in Table A5 are obtained by further fitting linear slopes to the PPAs in restricted regions around the SG points.

Figure 1 gives an example of the polarization displays and fits used throughout the paper. The upper panels show the usual average polarized profile information (Stokes $I, L$ and $V$ are given by solid black, dashed-red and dotted-blue curves, respectively), and the PPA is plotted twice in the lower panel for ease of viewing. As we will see below, our geometric analyses will often provide values for $\alpha$ and $\beta$, and the resulting RVM-based PPA traverses are indicated in the lower panel by a pair of dotted magneta curves. The light grey curve illustrates the effect of the above RVM-fitted parameters as obtained by fitting eq. 1 and given in Table A5. The "flared" total power profile is shown in the upper using a solid cyan curve (see $\S$ III text). 


\section{III. "Flared" emission}

Single pulses of pulsars show a great deal of variety. Generally, subpulses of varying intensity are seen to appear and disappear at various pulse longitudes, but when averaged together a stable pulse profile is formed. However, this is not always so: a few pulsars are known for their "giant" pulsesmost famously the Crab pulsar - and in a few others occasional bright pulses can be so very strong that the profile form is unstable (e.g., B0656+14; see Weltevrede et al 2006b). For a few other pulsars, "episodic" illumination has been observed that greatly emphasizes parts of a pulsar's profile at the expense of others (Rankin et al 2006a). For these reasons we thought it important to explore whether these effects could be active in some of L\&M's "partial cone" pulsars. We therefore undertook analyses similar to those of Hankins \& Cordes (1981) and Nowakowski (1991). Almost immediately, we discovered "flaring" effects in the single pulse emission of some of the "partial cone" pulsars.

In the lefthand panel of Figure 1 we show a GMRT total-power pulse sequence (hereafter PS) of the "partial cone" pulsar B0355+54 (pulse \#s 1800-1900). Notice that most of the bright emission occurs around $-10^{\circ}$ longitude (where zero longitude corresponds to the SG point of the PPA traverse); however, one strong subpulse can be seen extending to $+15^{\circ}$ (pulse \#1886) and several other fainter subpulses can be discerned around $-40^{\circ}$. Obviously, this pulsar shows great dynamicity in its pulse-to-pulse fluctuations: the core varies dramatically in intensity, often disappearing entirely; the leading and trailing conal outriding components are only occasionally detectable; and overall the pulsar nulls for some $30 \%$ of the time. These occasional "flares" of the conal components are then remarkable - and we find that they are very rare in B0355+54 - occurring only in 200 pulses within a PS of 13000 individual pulses.

We have searched for "flared" emission in the entire set of "partial cone" pulsars available to us. We used a "tunable" window to detect sporadic emission in the fainter regions of the average profile where the intensity is close to the noise level. Each time the emission exceeded three times the noise level (averaged over the window), we marked that pulse and window as having "flared" and with adjacent windows computed the average "flared" profile. We then repeated this process for different window sizes until the "flared" profile was stable over a range of window widths. The righthand display of Fig. 1 gives an example of this "flared"profile analysis for B0355+54, and the "flared" total power profile is shown using a solid cyan curve. Obviously, this "flared" profile shows the contributions of the sporadic emission to the far edges of the profile, and it strongly suggests a threecomponent structure.

We found evidence for "flaring" in about half the group of "partial cone" pulsars under study, and the full results are shown using displays similar to Fig. 1 in the Appendix. Overall, we found little difference between the widths of the "flared" profiles compared to the full discernible widths of the corresponding normal average profile; however, the structure was often more scrutable - and in some cases we used these "flared" widths in the geometric analyses given in Table A3, see the Appendix for discussions of the analyses of the individual pulsars. We also looked for periodicities in the "flares" and found no evidence for any regular repetitive behavior.

\section{Conal Profile Asymmetry and Symme- try}

Conal single pulsar B0809+74 was listed by $\mathrm{L} \& \mathrm{M}$ as a partial cone because of the strong evidence that its meter-wavelength profiles are asymmetric because they are incomplete - or "absorbed" (e.g., see Rankin et al 2006b), and we now know that a number of other conal single pulsars share this asymmetric property (e.g., B0943+10, see Deshpande \& Rankin 2001). Perhaps the asymmetry is due to the circumstance that stars with such profiles entail a highly tangential sightline traverse along the outer edges of their conal beams - but although we do not understand the cause of these asymmetries adequately, we do now know that nearly all conal single $\left(\mathbf{S}_{d}\right)$ profilesand many narrow inner-cone double $(\mathbf{D})$ profiles are asymmetric.

Perhaps then unsurprisingly, we found that a large proportion of the "partial cone" pulsars identified by L\&M had conal profiles that were either of the single $\left(\mathbf{S}_{d}\right)$ or unresolved double $(\mathbf{D})$ type. Many such pulsars exhibit regular drifting sub- 

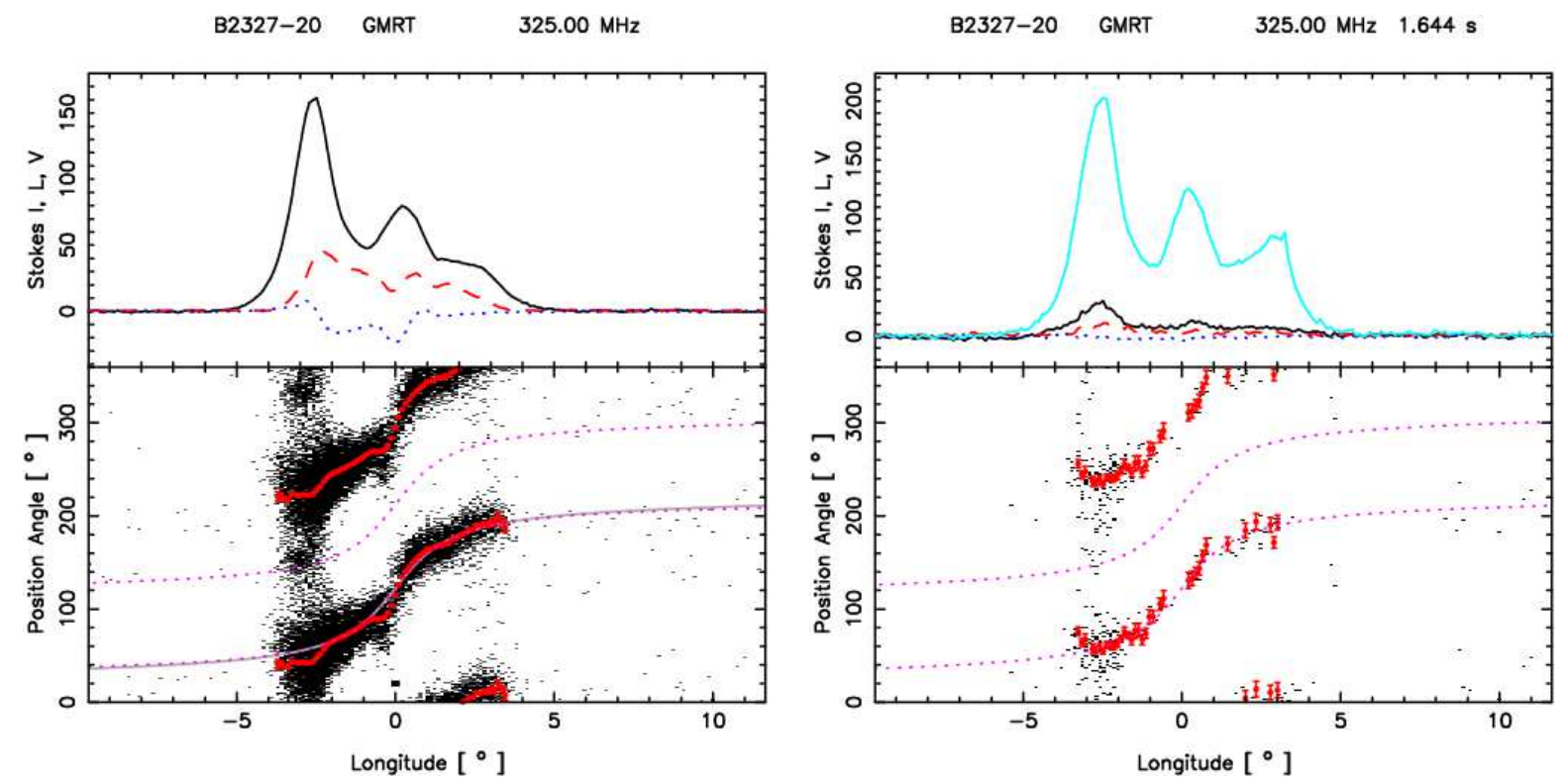

Fig. 3.- PPA histograms for pulsar B2327-20 as in Fig. 1 (right panel). The lefthand display shows the full PS, whereas the righthand plot includes only pulses having a lower intensity level. Notice that the latter PPA traverse is much smoother and can be used to fit the RVM. The longitude origin corresponds to the SG point of the PPA swing.

pulses and consequently show features indicative of periodic modulation in their fluctuation spectra. Some others, however do not - or do not all of the time (as in B0943+10's 'Q' mode) — but when such modulation can be detected it argues strongly for a conal association.

We therefore computed fluctuation spectra similar to those in Figure 2 for each of the PSs available to us. Most are not shown, usually because the same information was available in the published fluctuation-spectral compendia of Weltevrede et al (2006b, 2007; hereafter WES/WSE). Those dozen or so "partial cone" pulsars found to have $\mathbf{S}_{d}$ or $\mathbf{D}$ profiles - in a number of cases it was difficult to be sure which - are so denoted in Table A3, and their full analysis is discussed in the Appendix.

For a few other pulsars in our sample we found subpulse modulation features on both the leading and trailing edges of their profiles. Fig. 2 shows fluctuation spectra for pulsars B0740-28 and PSR B1910+20, where the same periodicity modulates both edges of their profiles in a stationary manner. If their subpulse "drift" is produced a carousel- beam system rotating about the magnetic axis, then this strongly indicates that the emission from these stars does indeed fill most of their polar fluxtube regions.

\section{The SG point and Profile Symmetry}

We have revisited these symmetry issues for all the "partial cone" pulsars for which we have high quality single pulse polarimetry. Our intent has been to determine whether the SG point of the PPA traverse, determined using RVM fitting, leads or lags the total intensity profile center. Single pulse polarimetry often helps to identify regions of OPM activity which otherwise can complicate the average-PPA traverse, and hence cause an inaccurate identification of the SG point. Thus, we have computed PPA histograms for each of the "partial cone" pulsars - which are reproduced in the Appendix - and wherever possible distinguished their separate PPA traverses before fitting the RVM to determine the SG point. In a few cases PS polarimetry was unnecessary to fix the SG point; however for pulsars like B1604-00 

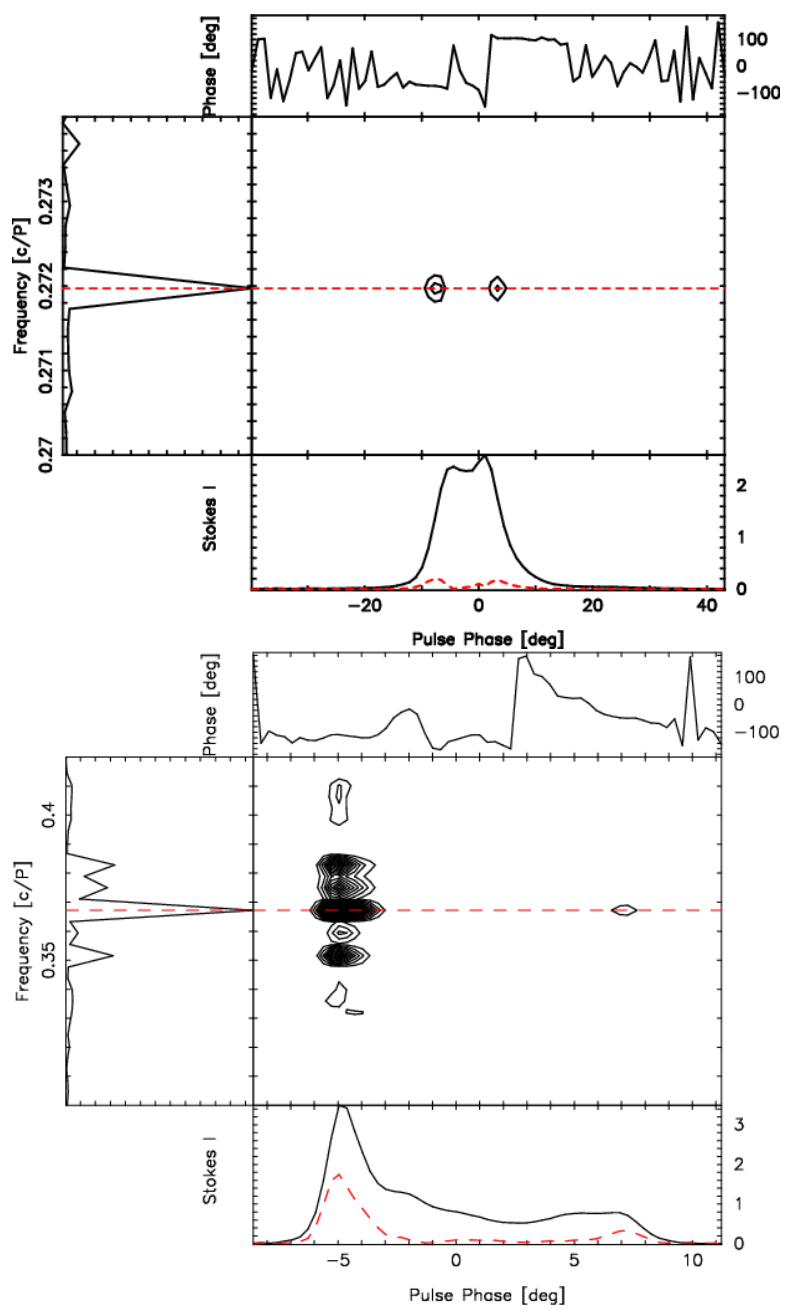

Fig. 2.- Fluctuation spectra for pulsars B074028 and B1910+20. The main panels show the fluctuation amplitudes in contours, and the longitudeaveraged aggregates are given in the lefthand panels. The bottom panels show the total-power average profiles, and the fluctuation phases are given in the top panels. or B2043-04 mode separation was essential before any sensible RVM fit could be made to their PPA traverses.

For several other pulsars, B1910+20 and B2327-20 among them, the average PPA traverse exhibited highly non-RVM behavior. Mitra et al (2007) noticed for pulsar B0329+54 that the PPA traverse can be intensity dependent, and we have used their technique of dividing the PS into intensity fractions in order to obtain a smooth PPA traverse. Figure 3 shows for pulsar B2327-20 how an apparently orderly RVM behavior can be recovered for fitting even when the total PPA profile is distorted by intensity effects. Here, the SG point obtained by fitting the RVM is well constrained. However, several pulsars in our sample (such as B0906-17, B1742-30 and B1112+50) show relatively flat PPA traverses, and hence the SG point is not well determined. Given all these various circumstances, we were able to obtain RVM fits for 24 pulsars. The fitted RVM parameters are listed in Table A5 and are used to plot the RVM as a grey line in the Figs. 1, 3] and many of those in the Appendix.

Of primary importance is the location of the $\mathrm{SG}$ point with respect to the overall extent of a pulsar's profile, so as to assess whether A/R is significant. We use the BCW method of finding the profile center-i.e., measuring the midway point between the outer $10 \%$-intensity points of the profile, and we then compare this with the location of the SG point. For the pulsars with SG points determined via RVM fits, we find that for all of them the SG point either trails or is consistant with fall at the midway point. Two apparent cases of leading SG points were quite interesting, B0138+59 and B2224+65: for the former early parts of the profile appear to be missing at all frequencies above 100 $\mathrm{MHz}$, but SVS's 102.5-MHz Faraday polarimetry shows it well centered; whereas for the latter, identification of the trailing component as a postcursor made any such argument untenable. Moreover, for several slow stars in the group, like B2043-04 and B2327-20, the SG point is consistent with being coincident with the profile center.

For several stars the SG point was not well determined and we lacked other observations of sufficiently high quality. The rightmost column of Table A2 then gives an overview of these SGpoint locations with respect the respective profile 
midpoints; here " $\mathrm{T}$ " refers to the case where the SG point leads the midway point, "L" (no cases) where the SG point trails, "U" refers to unclear cases where the midway or the SG point determination fails, and "_-" to those cases where the profile center is consistent with being coincident with the SG point. Justification for the SG point determinations for individual stars are found in the Appendix.

\section{Abberation/Retardation Effects}

We saw just above that the PPA-traverse SG points falling on or after the profile centers all the cases. A natural explanation for this circumstance is the abberation/retardation $(\mathrm{A} / \mathrm{R})$ effects first studied by Blaskeiwicz et al (1991; BCW). This BCW model provided a substantial improvement over the RVM model, by incorporating these $(\mathrm{A} / \mathrm{R})$ relativistic effects on pulsar-emission properties. For emission arising from a finite height $r_{e m}$ above the center of a rotating neutron star with period $P_{1}$, they derived an expression for the PPA $\chi$ as a function of pulse longitude $\varphi$ as,

$\chi=\tan ^{-1}\left(\frac{\sin \alpha \sin \left(\varphi-\varphi_{\circ}\right)-3 \hat{r} \sin \xi}{\sin \xi \cos \alpha+\cos \xi \sin \alpha \cos \left(\varphi-\varphi_{\circ}\right)}\right)+\chi_{\circ}$

where $\hat{r}=r_{e m} / r_{c}$ is the emission height $r_{e m}$ in terms of the light-cylinder radius $r_{c}=c P_{1} / 2 \pi$, and $c$ is the speed of light. Note that eq. 2 reduces to the RVM (eq. 1) for $r_{e m}$ tending to zero.

In short, the BCW model predicts that for radio emission arising from a constant finite height, the overall PPA traverse will lag the total intensity profile. To first order, particularly for slowly rotating pulsars, this shift is a simple translation of the PPA traverse towards the trailing parts of the profile-hence no change is required to fit the RVM to the PPA traverse. However, the SG point will now be found to lag the profile center by an amount $\Delta \varphi=4 \pi r_{e m} / P_{1} c$, a shift which has been observed in several pulsars (e.g., BCW, von Hoensbroech \& Xilouris 1997, Mitra \& Li 2004) and then used to estimate the relevant radio emission heights - giving typical values of a few hundred kilometers. In some studies, (see e.g. Malov \& Suleimanova 1998; Gangadhara \& Gupta 2001, Krzeszowski et al 2009), Srostlik \& Rankin(2005) and Force \& Rankin (2010) this shift is also seen with respect to the central core component.
Here we want to justify our conclusion that for the majority of "partial cone" pulsars, the lagging of the SG point with respect to the profile center is primarily due to $\mathrm{A} / \mathrm{R}$ effects. On the one hand, the "flared" profile analysis provides a valuable method of assessing the full emission width in longitude - that is, the total extent of emission activity within the polar flux-tube region - and this in turn permits us to be more certain about the position of a profile's center and thus the relative placement of the SG point. Then, on the other hand, the RVM fitting often permits us to be sure about the symmetry properties of the PPA traverse and thus its placement relative to the profile center. The shift $\Delta \varphi$ is hence found as the difference between the (conal component-pair) profile center and the SG point, and it is from this quantity that an emission height can be computed.

Among our "partial cone" population, we found 13 cases for which an $\mathrm{A} / \mathrm{R}$ emission height could be computed as above, and these are tabulated in Table A4. For two stars B1732-07 and B174230 we have used the central core-component peak with respect to which the shifts have been computed for finding the emission heights. Note that the values obtained are roughly $200-400 \mathrm{~km}-$ therefore, not very different from those height estimates computed for normal (non- "partial cone") pulsars. This result strongly supports the conclusion that the majority of "partial cone" pulsars are very similar to other "normal" pulsars - that is, their emission arises from similar heights and (at least sometimes) involves most of the polar cap region. For the few slower pulsars in the "partial cone" population, PSR B1910+20, B2043-04 and B2327-20, the measured A/R shifts are small, such that the profile centers and SG points are almost coincident. Although one expects that A/R shifts should be inversely related to pulsar period, such that faster pulsars should show larger shifts, none of the $\mathrm{A} / \mathrm{R}$ studies in the literature has cleanly demonstrated this effect. Failure to see this effect systematically could be due to a number of factors [see the detailed discussion by Mitra\& Li (2004) on factors affecting A/R effects].

We note that similar effects have been found by Karastergiou \& Johnston (2006) in B1054-62 and B1356-60, the latter of which is discussed below in the Appendix with the other "partial cones". Several other cases where A/R appears to affect 
the profile structure are denoted by "ar" in their Table A3 classifications.

\section{Aberrant Linear Polarization Signa- tures?}

Our recent analyses (Backus et al 2010) on the precursor components of pulsars B0943+10 and B1822-09, strongly suggest that these features are "other" - that is, they are not emitted at low altitude in the polar flux tube as is the conal and core emission with which we are familiar. We argued that the precursors were aberrant largely on the basis of their nearly complete linear polarization and flat PPA traverses. Among L\&M's "partial cone" grouping, we encounter B1822-09 again, and the geometric analysis in Table A3 (see also Fig. A6 reflects the conclusions of the above study in that we do not regard the star's precursor component as a part of its main pulse.

Three other such objects were found among L\&M's "partial cones", B1322+83, B1530+27 and B2224+65. In the first case seen in Fig. A3 the highly polarized feature is a precursor to what otherwise is probably a conal single main pulse; whereas, for the latter two in Fig. A3 and A10 the aberrant features fall as a postcursors to what seem to be a conal single and core-single main pulses, respectively. A number of other such features can now been found in the published polarimetry, but at the time of L\&M's study, very few were known, so it is not surprising that they regarded them as outstanding in core/core terms. Indeed, they yet remain so, but we now know of enough that they represent something of a distinct phenomenon.

For B1322+83, we note also that were this star an asymmetric conal double (which is not what we conclude), the putative profile midpoint at about $-4^{\circ}$ falls far ahead of the SG point under the trailing feature. Following this interpretation we can compute an A/R emission height of some $3700 \mathrm{~km}$, which is very large for any pulsar. Therefore this interpretation is almost certainly incorrect.

\section{Analyses of the Emission Geometry}

Paper VI of this series gave an extensive analysis of the emission geometry of some 200 pulsars. The core-component width $W_{\text {core }}$ was often used to determine the magnetic latitude $\alpha$ using the relationship $W_{\text {core }}=P_{1}^{-1 / 2} / \sin \alpha$ (Paper IV). The sightline impact angle $\beta$ could then be fixed (within a sign 3 ) using $\alpha$ and the PPA sweep rate $R$, which can be determined empirically at the SG point as $|\Delta \chi / \Delta \varphi|_{o}$ (and within the RVM is also $\sin (\alpha) / \sin (\beta)$; see eq. 11). Finally, the conal radii were computed using the dimensions of the conal components or pairs as in Paper VI; see eqs. (2-6). Several of the "partial cone" pulsars under study here were also included in this $\mathrm{Pa}$ per VI analysis, but the results-based entirely on average-profile dimensions - were disappointing just as they were for L\&M and for virtually the same reasons. Detailed geometric models for a few others have been developed elsewhere; see Table A3, footnotes b-f.

Here we are now in a position to reinvestigate the emission geometry of L\&M's "partial cone" population with much more information and thus a greatly enhanced expectation of success. The pulsar-by-pulsar discussions in the Appendix together with Table A5 summarize the RVM-fitting results for all those stars for which it was possible, and Table $\mathrm{A} 3$ gives the PPA sweep rate $R$ in boldface when determined by this fitting. Similarly, this table shows $\alpha$ in boldface when it was possible to estimate it from a core width or by other means. Then, the conal dimensions are computed from the profile width information just as was done previously in Paper VI. The outside halfpower widths of the respective inner and outer conal beams are given along with the estimated emission heights - and the parameter $\beta / \rho$ is also tabulated for many pulsars as an indication of the expected profile form.

The overwhelming results of Paper VI, confirmed by later studies, is that pulsar emission cones come in two types, inner and outer, with outside half-power 1-GHz dimensions of 4.33 and $5.75^{\circ}$, respectively. Somewhat surprisingly, this implies nominal $1-\mathrm{GHz}$ emission heights of some 130 and $220 \mathrm{~km}$, respectively. Therefore, just as in Paper VI, we here endeavor to demonstrate two distinct propositions: a) when all the above information is available, we show that a specific "partial cone" pulsar will have compatible conal radii

\footnotetext{
${ }^{3}$ All have been taken positive, as the poleward or equatorward sense of the sightline traverse cannot easily be known. In fact, the sense of $\beta$ usually makes little difference in these modeling computations.
} 


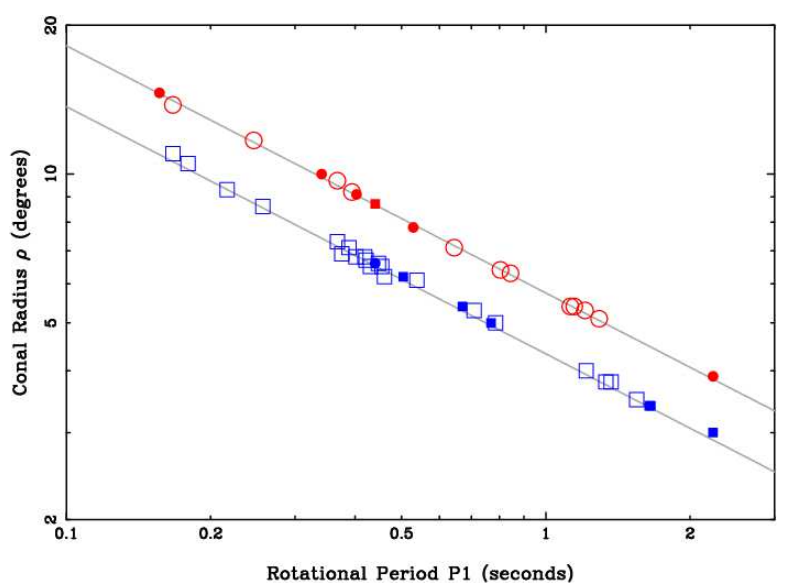

Fig. 4.- Plot showing (outside half-power) conal emission radius $\rho$ vs. rotation period $P_{1}$ for the "partial cone" population of pulsars in Table A3. The red symbols represent the outer cones and the blue the inner cones, respectively. The filled symbols reflect a full analysis where the magnetic latitude $\alpha$ can be determined from the core width and $\beta$ then determined from the fitted PPA sweep rate $R$; whereas, the open symbols indicate pulsars for which compatible geometrical configurations could be identified despite having no means of estimating $\alpha$ independently — all as in Paper VI (see text for details). The lower and the upper grey lines correspond to the characteristic inner and outer conal radii of $4.33 P_{1}^{-1 / 2}$ and $5.75 P_{1}^{-1 / 2}$, respectively and thus nominal emission heights; however, b) when this "full solution" is impossible, we use the available information to model the emission geometry to achieve appropriate radii and heights, thus resulting in a useful estimate of the magnetic latitude $\alpha$. When multifrequency profiles are available, it is usually clear whether a specific star has an inner or outer cone (or in a few cases both) because outer cones spread strongly with wavelength and inner ones do not. Finally, we have taken $\beta$ positive in all cases, given that its sign cannot usually be determined.

One might worry that the conal dimension and emission heights for pulsars in category b) above are meaningless because the former have been constrained to values near the characteristic inner or outer conal radius. However, this exercise is hardly arbitrary: Paper VI showed that there were two distinct types of cones, inner and outer, with specific angular dimensions and therefore nominal emission heights, and other work (e.g., Paper VII) demonstrated that the two conal species could usually be distinguished by whether or not their dimensions increased significantly at low frequency. For each such pulsar we have used all available evidence to make this determination (as discussed for each star in the Appendix), and when successful - as it was in most cases - appropriate angular radii could be determined. Then, we explored whether a value of $\alpha$ existed that was compatible with each star's conal radius together with its profile's conal width and PPA sweep rate $R$ (so as to determine $\beta$ ) using Paper VI: eq.(4). Usually such an $\alpha$ could be found providing a plausible geometric model, and these values are given in Table A3 and the conal radii plotted as the open symbols in Figure 44 Such simple models have proven to be quite reliable-e.g., as many of the values in Bhattacharya \& van den Heuvel's (1991) comparison were estimated by this means.

For several reasons the geometrical error indications in Table A3 are only approximate. Our purpose here is to demonstrate the overall geometric "normality" of L\&M's "partial cone" population. A definitive geometric analysis of the individual stars is far beyond the possible scope of this ef-

\footnotetext{
${ }^{4}$ Note that the geometrical assumptions behind eq.(4) above are only satisfied within certain parameter ranges, and in a few cases (i.e., B1930+22) no model could be constructed despite some suggestion of a core/cone structure.
} 
fort. Often, for instance, no pair of quality high and low frequency observations was available, so we could not extrapolate to $1 \mathrm{GHz}$ as in Paper VI. More importantly, the difficult character of this population has entailed using a variety of methods with different assumptions and uncertainties. Therefore, where an $R$ value could be determined by fitting, we show it in bold in Table A3 with its errors given in Table $\mathrm{A5}$, and where an $\alpha$ could be estimated by a core width, it is also so indicated by bold type. The conal dimensions in Table A3 are typically accurate to about a degree or so of longitude; when they could be measured more precisely (and extrapolated to $1 \mathrm{GHz}$ ), we give them with a decimal, or when very approximate, we show them with a question mark. Finally, when the conal radii and emission heights could be fully determined, the latter are shown in bold. These values near 130 and $220 \mathrm{~km}$ are only nominal, rather than physical, quantities, so the accuracy of their determination is of secondary importance.

These geometric results are then plotted in Fig. 4, and the results are quite dramatic. The values fall on two parallel tracks representing the outer and inner conal radii, respectively. The solid symbols indicate the full solutions above, and the open ones compatibility where $\alpha$ could not be determined independently. Overall we see that there is no geometric distinction at all between L\&M's "partial cone" pulsars and those with more ordinary and symmetrical profiles. Of course, all of the "partial cone" pulsars for which we have observations are not represented in Fig. 4- some of them are very difficult to understand as we have seen in the previous section - but here we see clearly that the great majority exhibit the same orderly conal and core dimensions as was found earlier in Paper VI.

\section{Summary and Conclusions}

In the foregoing sections we restudied the population of "partial cone" pulsars so identified by Lyne \& Manchester in 1988. Using a combination of recent GMRT and Arecibo polarimetry, we have based our analyses predominantly on sensitive PS observations. These analyses have attempted to identify regions of "flared" and A/Red emission as well as searching for the more usual periodic subpulse modulation.
Through this analysis we have been able to show that-

- In general, L\&M's "partial cone" pulsars exhibit no particular property or difference as compared to the rest of the slow pulsar population. Rather, they exhibit a range of characteristics, many of which are well understood and some of which are not.

- Overall the "partial cones" exhibit cone and double cone profile structures just as the "normal" pulsars do. To a significant extent PS analyses are needed to establish this regularity, because many of the "partial cones" do preferentially illuminate only a part of their polar flux-tube emission regions. However, when these small difficulties are accounted for, the emission geometry of most "partial cones" is remarkably regular in the terms established in Paper VI - that is, both the cores and cones have particular angular dimensions that scale with the size of a pulsar's polar cap.

- We find several further examples among the "partial cones" of highly polarized pre- or postcursors with flat PPA traverses. Following our analysis of such features in B0943+10 and B1822-09 (Backus et al 2010), we argued that these features cannot be emitted at low altitude within the usual polar fluxtube region. Such features are important, because they provide clues to the electrodynamics of the larger magnetosphere. Clearly this emission is coherent (highly polarized), beamed and likely emitted at very high altitude.

- Among the "partial cone" pulsars we find no good examples of profiles where the SG point leads the profile center. Surely this can be taken as strong evidence that $\mathrm{A} / \mathrm{R}$ operates to some degree in all pulsar profiles.

- A number of examples of A/R shifts, both in the PPA traverses and component positions, were encountered among the "partial cones". It seems likely that $\mathrm{A} / \mathrm{R}$ is an important factor in distorting the core/cone structure of pulsars that rotate quite rapidly. 
It is hardly surprising that a study as ambitous at that of Lyne \& Manchester's would encounter a residuum of objects that were difficult to categorize and study. Indeed, the "partial cone" population were overall among the faster, weaker and more highly dispersed stars available to them in 1988. A variety of both technical and scientific factors now permit us to understand that most of these "partial cone" pulsars are as "normal" in their beaming geometry as the many studied in earlier papers in this series. Technically, quality PS polarimetry of highly dispersed stars with periods down to $100 \mathrm{msec}$ are no longer challenging. Scientifically, a great deal has been learned about cones and their constituent subbeam carouselsand that the emission from such systems rarely produces symmetrical profiles. Single pulse observations permit us to identify emission centers that are too weak or irregular to show in up in average profiles. Similarly, BCW introduced the A/R discussion only three years after L\&M's study, but it has taken fully these two decades for workers to begin to identify $\mathrm{A} / \mathrm{R}$ regularly and confidently in pulsar emission, given the many other factors that tend to obscure its full effect. Finally, we now see that the "normal" core/cone emission from the slow pulsar population is regular enough in its properties that some aberrant features can be identified. We cannot yet be sure where and how the highly polarized pre- and postcursor features are emitted, but it seems ever less likely that they come from the usual low altitude polar fluxtube region.

The other effect that $\mathrm{A} / \mathrm{R}$ predicts - that the intensity of the leading conal regions of the profile will be brighter than the trailing parts - this we do not see in our analyses. Rather it appears that the probability of radio emission across the pulse profile (or within the polar flux tube) varies strongly. For example, the "flared" emission we see in several stars (e.g., PSR B0355+54 in Fig. 1) is overall rare, occurring within only $1-5 \%$ of all active pulse longitudes, whereas in many other such regions the emission is virtually continuous, occurring essentially $100 \%$ of the time. Of course, this implies that the shape of a pulsar's total-intensity profile varies strongly across the "active" window because the several different processes entailed in this emission also vary strong with longitude. The PPA traverse, on the other hand, closely follows the RVM (particularly when complications due to OPM and A/R effects can be accounted for).

Hence, the phenomenological model that emerges from our overall analyses is that pulsar coherent radio emission almost always arises from open dipolar field lines, at several hundred $\mathrm{km}$ above the pulsar polar cap. Within this polar flux-tube region, there is an underlying double cone/core structure of the pulsar radio-emission beams, although the pulse shape itself depends on the probability of coherent radio emission $\left(P_{c r e}\right)$, which varies strongly with magnetic colatitude and azimuth and thus with pulse longitude along the sightline trajectory of a given star and viewing geometry. For example, under the vacuum-gap model and assuming curvature radiation as the radio emission mechanism, $P_{c r e}$ should be viewed as a combined probability of $P_{c r e}=P_{p p c} \times P_{s p c} \times P_{c c r}$. Here $P_{p p c}$ is the probability of primary pair creation in the vacuum gap, $P_{s p c}$ is the probability of secondary pair creation and $P_{c c r}$ would be the criteria for exciting coherent curvature radiation (RS75, Sturrock 1971, Gil, Lyubarsky \& Melikidze 2004). Further since average pulse profiles are stable, $P_{\text {cre }}$ fluctuates around a mean value, indicating the presence of a stable physical quantity at every pulse longitude. We conjecture that $P_{c r e}$ is primarily guided by the underlying multipolar magnetic field across the polar cap. Such a structure causes the field to vary in magnitude and curvature radius across the polar cap (see Gil, Melikidze \& Mitra 2002). The field at the region where radio emission arises is however significantly dipolar.

We thank our anonymous referee and the editor for critical comments which helped in improving the manuscript significantly. We are pleased to acknowledge S. Sarala for important assistance with aspects of the observations and analysis of GMRT data. We thank our first referee Aris Karastergiou for encouraging us to enlarge our study to include as many of L\&M's "partial-cone" pulsars as possible. We thank the staff of the GMRT that made these observations possible. We also wish to thank Joel Weisberg for his assistance with the ionospheric Faraday rotation corrections. One of us (JMR) thanks the Anton Pannekoek Astronomical Institute of the University of Amsterdam for their generous hospitality and both Netherlands 
National Science Foundation and ASTRON for their Visitor Grants. DM thanks National Astronomy and Ionosphere Center, Arecibo Observatory, Puerto Rico, for supporting a visiting scientist position during which this work was completed. Portions of this work were carried out with support from US National Science Foundation Grants AST 99-87654 and 08-07691. Arecibo Observatory is operated by Cornell University under contract to the US NSF. GMRT is run by the National Centre for Radio Astrophysics of the Tata Institute of Fundamental Research. This work used the NASA ADS system.

\section{REFERENCES}

Backer D.C. 1976, Ap.J., 209, 895

Backus I., Mitra, D., \& Rankin, J. M., 2010, M.N.R.A.S., 404, 30. (BMR)

Bartel, N., Kardeshev, N. S., Kuzmin, A D., Nikolaev, N.Ya., Popov, M.V., Sieber, W., Smirnova, T.V., Soglasnov, V.A., \& Wielebinski, R. 1981, A\&A, 93, 85.

Bartel, N. 1981, A\&A, 97, 384.

Bhattacharya, D. \& van den Heuvel, E.P.J. 1991, Physics Reports, 203, 1-124

Biggs, J. D., 1990, M.N.R.A.S., 246, 341.

Blaskiewcz, M., Cordes, J.M., \& Wassermann, I. 1991 Ap.J., 370643 (BCW)

Costa, M. E., McCulloch, P. M., \& Hamilton, P. A. 1991, M.N.R.A.S., 252, $13(\mathrm{CMH})$

Deshpande, A. A. \& Rankin, J., 2001, M.N.R.A.S., 322,438

Deich, W.T.S. 1986, M.S. Thesis, Cornell Univ. (D86)

Deich, W.T.S., Cordes, J. M., Hankins, T. H., \& Rankin, J. M. 1986, Ap.J., 300, 540 (DCHR)

Downs, G. S. 1979, Ap.J. Suppl., 40365

Dyks, J., Rudak, B. \& Harding, A. K., 2004, Ap.J., 607, 939

Everett, J. E., \& Weisberg, J. M. 2001, Ap.J., 553, 341
Force, M. M., \& Rankin, J. M. 2010, M.N.R.A.S., 406, 237

Gangadhara, R.T., \& Gupta, Y. 2001, Ap.J., 555, 31

Gil, J.A., Kijak, J., \& Seiradakis, J.H. 1993, A\&A, 272,268

Gil, J.A, Lyne, A.G., Rankin, J.M., Snakowski, J.K., \& Stinebring, D.R. 1992, A\&A, 255, 181

Gil, J. A., \& Lyne, A. G. 1995, M.N.R.A.S., 276, 55.

Gil, J. A., Melikidze, G. I., \& Mitra, D. 2002, A\&A, 388, 246.

Gil, J. A., Lyubarsky, Y., \& Melikidze, G. I. 2004, Ap.J., 600, 872.

Gould, M. \& Lyne, A, 1998, M.N.R.A.S., 301, 235 (GL)

Gupta, Y., \& Gangadhara, R.T. 2003, Ap.J., 584, 418

Gupta, Y.; Gothoskar, P., Joshi, B. C., Vivekanand, M.; Swain, R., Sirothia, S.\& Bhat, N. D. R., 2000, ASP Conf. Ser. 202: IAU Colloq. 177: Pulsar Astronomy - 2000 and Beyond, 227

Hamilton, P. A., McCulloch, P. M., Ables, J. G., \& Komesaroff, M. M. 1977, M.N.R.A.S., 180, 1 (HMAK)

Hankins, T. H., \& Cordes, J. M. 1981, Ap.J., 249, 241

Hankins, T. H., \& Rankin, J. M. 2010, A.J., 139, 168

Hankins, T. H., \& Wolszczan, A. 1987, Ap.J., 318, $410(\mathrm{HW})$

von Hoensbroech, A., \& Xilouris K. 1997, A\&A Suppl., 126, 121 (vHX)

von Hoensbroech, A. 1999, Ph.D. thesis, Univ. of Bonn (vH)

Johnston, S., Hobbs, G., Vigeland, S., Kramer, M., Weisberg, J. M., \& Lyne, A. G. 2005, M.N.R.A.S., 364, 1397 (J-05) 
Johnston, S., Karastergiou, A. \& Willett, K. 2006, M.N.R.A.S., 369, 1916 (JKW)

Johnston, S., Kramer, M., Karastergiou, A., Hobbs, G., Ord, S. \& Wallman, J. 2007, M.N.R.A.S., 381, 1625 (J-07)

Johnston, S., Karastergiou, A., Mitra, D \& Gupta, Y, 2008, M.N.R.A.S., 388, 261

Karastergiou, A. \& Johnston, S. 2006, M.N.R.A.S., 365, 353 (KJ)

Karastergiou, A. \& Johnston, S. 2007, M.N.R.A.S., 380, 1678

Kloumann, I. M., \& Rankin, J. M. 2010, M.N.R.A.S., 408, 40 (KL10)

Komesaroff, M.M. 1970, Nature, 225, 612

Kramer, M. 1994, A\&A Suppl.107, 527

Krzeszowski, K., Mitra, D., Gupta, Y., Kijak, J., Gil, J. \& Acharyya, A., 2009, M.N.R.A.S., 393, 1617

Kuzmin, A. D., Izvekova, V. A., Shitov, Yu. P., Sieber, W., Jessner, A., Wielebinski, R., Lyne, A. G., \& Smith, F. G. 1998, A\&A Suppl., 127, 355 (K-98)

Kuz'min, A. D. \& Losovskii, B. Y. 1999, Astr. Repts., 43, 288 (KL)

Lyne, A.G. 1990, private communication (L90)

Lyne, A.G., \& Manchester, R.N. 1988, M.N.R.A.S., 234, 477 (LM)

Malofeev, V. M., Izvekova, V. A., \& Shitov, Yu. P. 1986, preprint FIAN USSR (MIS)

Malov, O. I., \& Malofeev, V. M. 2010, Astronomy Rpts., 54, 210 (MM)

Malov, I. F., \& Suleimanova, S. A. 1998, Astr. Rpts. 42,388

Manchester, R.N., Hamilton, P.A., \& McCulloch, P.M. 1980, M.N.R.A.S., 192, 153 (MHM)

Manchester, R.N., Han, J.K., \& Qiao, G.J. 1998, M.N.R.A.S., 295, 280 (MHQ)

McCulloch, P.M., Hamilton, P.A., Manchester, R.N., \& Ables, J.G. 1978, M.N.R.A.S., 183, 645 (MHMA)
McCulloch, P.M., Hamilton, P.A., \& Manchester, R.N. 1982, private communication (MHMb)

Mitra D \& Deshpande A. A., 1999 A\&A, 346, 906

Mitra, D, Gupta, Y. \& Kudale, S., 2005, "Polarization Calibration of the Phased Array Mode of the GMRT", URSI GA 2005, Commission J03a

Mitra, D. \& Rankin, J. M. 2002, Ap.J., 557, 322 (Paper VII)

Mitra, D., \& Li, X. H., 2004, A\&A, 421, 215

Mitra, D., Rankin, J. M. \& Gupta, Y. 2007, M.N.R.A.S., 379, 932

Morris, D., Sieber, W., Ferguson, D. C. \& Bartel, N., 1980, A\&A, 260, 262

Morris, D., Graham, D.A., Sieber, W., Bartel, N., \& Thomasson, P. 1981, A\&A, 46, 421 (MGSBT)

Nowakowski, L. A. 1991, Ap.J., 377, 581.

van Ommen, T.D., D'Alessandro, F., Hamilton, P.A., \& McCulloch, P.M. 1997, M.N.R.A.S., 287, 307 (vO97)

Radhakrishnan, V., \& Cooke, D. J. 1969, Ap. Lett, 3,225

Radhakrishnan, V., \& Rankin, J. M., 1990, Ap.J., 352,258

Ramachandran, R., Backer, D.C., Rankin, J.M., Weisberg, J.M., \& Devine, K.E. 2004, Ap.J., 606,1167

Rankin, J.M. 1983a, Ap.J., 274333 (Paper I)

Rankin, J.M. 1983b, Ap.J., 274359 (Paper II)

Rankin, J.M. 1986, Ap.J., 301, 901 (Paper III)

Rankin, J.M. 1988, Ap.J., 325, 314

Rankin, J.M. 1990,Ap.J., 352, 247 (Paper IV)

Rankin, J.M. 1993, Ap.J., 405, 285 and A\&A Suppl., 85, 145 (Paper VI)

Rankin, J.M. \& Ramachandran, R., 2003, Ap.J., 590, 411 (Paper VIII) 
Rankin, J.M., Rodriguez, C., \& Wright, G.A.E. 2006a, M.N.R.A.S., 370, 673 (RRW)

Rankin, J.M., Ramachandran, R., \& Suleymanova, S.A. 2006b, A\&A, 447, 235 (RRS)

Rankin, J.M., Ramachandran, R., van Leeuwen, J., \& Suleymanova, S.A. 2006c, A\&A, 455, 215 (RRvLS)

Rankin, J. M., Stinebring, D. R., \& Weisberg, J. M. 1989, Ap.J., 346, 869 (RSW)

Ruderman, M.A. \& Sutherland, P.G. 1975, Ap.J., 196,51

Seiradakis, J. M., Gil, J. A., Graham, D. A., Jessner, A., Kramer, M., Malofeev, V. M., Sieber, W., \& Wielebinski, R. 1995, aaps, 111, 205. (S95)

Sturrock, P. A. 1971, Ap.J., 164, 529.

Suleymanova, S. A., Volodin, Yu. V., \& Shitov, Yu. P. 1988, Astro. Zh., 422, 17 (SVS)

Suleymanova, S. A., \& Shitov, Yu. P. 1994, Ap.J., 422, $17(\mathrm{SS})$

Sirothia, S. 2000, M.Sc. thesis, University of Pune

Srostlik, Z., \& Rankin, J. M. 2005, M.N.R.A.S., 362,1121

Stairs, I. H., Thorsett, S. E. \& Camilo, F., 1999, Ap.J., 123, 627

Swarup, G., Ananthakrishnan, S., Kapahi, V. K., Rao, A. P., Subrahmanya, C. R., Kulkarni, \& V. K. 1991, Current Science 60, 95.

Weisberg, J. M., Cordes, J. M., Lundgren, S. C., Dawson, B. R., Despotes, J. T., Morgan, J. J., Weitz, K. A., Zink, E. C., \& Backer, D. C. 1999, Ap.J. Suppl., 121, 171 (W-99)

Weisberg, J. M., Cordes, J. M., Kuan, B., Devine, J. E., Green, J. T., \& Backer, D. C. 2004, Ap.J. Suppl., 150, 317 (W-04)

Weltevrede, P., Edwards, R. T., \& Stappers, B. W. 2007, A\&A, 469, 607 (WES)

Weltevrede, P., Stappers, B. W., \& Edwards, R. T. 2006b, A\&A, 445, 243 (WSE)
Weltevrede, P., Wright, G.A.E., Stappers, B. W., Rankin, J. M. 2006a, A\&A, 458, 269 (WWSR)

Weltevrede, P., \& Wright, G.A.E. 2009, M.N.R.A.S., 395, 2117 (WW09)

Wu, X.J., Manchester, R.N., Lyne, A.G., \& Qiao, G.J. 1993, M.N.R.A.S., 261, 630 (WMLQ)

Xilouris, K.M., Rankin, J.M., Seiradakis, J.M., \& Sieber, W. 1991, A\&A, 241, 87 (XRSS)

Xilouris, K. M., Seiradakis, J. H., Gil, J., Sieber, W., \& Wielebinski, R. 1995, A\&A, 293, 153 (X95)

Xilouris, K. M., Kramer, M., Jessner, A., von Hoensbroech, A., Lorimer D, Wielebinski, R., Wolszczan, A. \& Camilo, F., 1998, Ap.J., 501, 286

The following is a list of the pulsars mentioned to comply with $\mathrm{AAST}_{\mathrm{E}} \mathrm{X} \S 2.15 .3$. It doesn't print in the right place. B0138+59 B0254-53 $\mathrm{B} 0355+54 \mathrm{~B} 0450+55 \mathrm{~B} 0540+23 \mathrm{~B} 0643+80 \mathrm{~B} 0740-28$ B0809+74 B0906-17 B0919+06 B1055-52 B1112+50 B1221-63 B1240-64 B1322+83 B1356-60 B1426-66 B1449-64 B1530+27 B1530-53 B1540-06 B1556-44 B1604-00 B1612+07 B1641-45 B1648-42 B1700-18 B1732-07 B1742-30 B1745-12 B1756-22 B1822-09 B1842+14 B1851-14 B1859+07 B1900+05 B1907-03 $\mathrm{B} 1910+20 \mathrm{~B} 1913+10 \mathrm{~B} 1915+13 \mathrm{~B} 1924+16 \mathrm{~B} 1930+22$ B1937-26 B1944+17 B1944+22 B2021+51 B2043-04 $\mathrm{B} 2053+36 \mathrm{~B} 2217+47 \mathrm{~B} 2224+65$ B2327-20

This 2-column preprint was prepared with the AAS IATEX macros v5.2. 


\section{APPENDIX: Results for Individual Pulsars}

Table 2 Notes: Pulsars with Bnames in normal type appear in L\&M's Table 4; those in italics are denoted as "Partial cones?" in their Table 5; and one other star, B1859+07 (parentheses) is included from our own work. The periods $\left(P_{1}\right)$, age $\left(\tau=P_{1} / 2 \dot{P}_{1}\right)$, magnetic field $(B)$ and energy are taken from the ATNF pulsar catalogue. The referenced figures appear in the Appendix, and the last column specifies if the SG point is either trailing $(\mathrm{T})$ or leading (L) or unclear (U) wrt the pulsar profile, and the (-) refers to cases where the SG point is consistent with being coincident with the profile center.

Table 3 Notes: Pulsars with Bnames in normal type appear in L\&M's table 4; those in italics are denoted as "Partial cones?" in their Table 5; and one other star, B1859+07 (parentheses) is included from our own work. The $\alpha$ values in boldface were determined using the corewidth method; while the others were estimated from profile dimensions. The $|\Delta \chi / \Delta \varphi|_{o}$ values in boldface were determined by PPA fitting; the others were taken from Paper VI or the ${ }^{a}$ values from L\&M. Other geometric solutions as follows: ${ }^{b} \mathrm{RRS} / \mathrm{RRvLS} ;{ }^{c} \mathrm{RRW} ;{ }^{d} \mathrm{WW} 09 ;{ }^{e} \mathrm{BMR} ;{ }^{f} \mathrm{KL} 10$.

B0138+59 presents an excellent example of a partial-cone profile in LM's intended sense. As in the $325 \mathrm{MHz}$ PS in Figure A1 and LM:fig. 6, we mainly see the central and trailing parts of what could be a conal double $(\mathbf{M})$ or quadruple $(\mathbf{Q})$ structure. Only at $100 \mathrm{MHz}$ does the leading feature fully reveal itself in the Pushchino profiles (SVS, MIS, K-98, KL), making the full half-power width nearly $40^{\circ}$. This suggests an outer cone with a 1-GHz width of about $27^{\circ}$, and a core width can be estimated as some $6-7^{\circ}$ constraining $\alpha$ to some $20^{\circ}$. Moreover, SVS's elegant $102.5-\mathrm{MHz}$ Faraday polarimetry clearly counters any easy argument that this star's SG point leads its profile center; the star's leading profile region is not visible at higher frequencies, but here the SG point lies near the profile midpoint.

The PPA traverse using the GMRT PS at 325 $\mathrm{MHz}$ is fitted with the RVM to obtain the SG point (see Table A5) which is well constrained by the PPA fit is taken as the zero longitude in Figure A1. We see no flared emission towards the profile edges; however the fluctuation spectrum shows a low frequency excess as has been reported by the WES/WSE analyses. Given that the existing $100-\mathrm{MHz}$ profiles seem to reveal the bright leading feature, sensitive new observations at low frequen- cies are needed to investigate this missing area of emission.

B0254-53 seems to have a narrow, conal double (D) profile (MHM, MHMA, MHMb, MHQ, vO97) with a slightly stronger leading component above 1 $\mathrm{GHz}$ and the reverse below. Its profiles are nearly depolarized and the PPA information difficult to interpret. The sweep value given by L\&M seems too steep; rather we use a value of $-8^{\circ} /{ }^{\circ}$ from the $278-\mathrm{MHz} \mathrm{MHMb}$ profile. In short, it is not clear why L\&M regarded this pulsar as a "partial cone". PSR B0355+54: The pulse profile at various frequencies (e.g., LM, GL, Xilouris et al 1998) clearly show three components, and the pulsar is classified as a core single by R93 due to the domination of the bright central component over the weak conal outriders. L\&M identifies this pulsar as a "partial cone" owing to its asymmetric profile at high frequencies with the SG point of PPA traverse lying towards the trailing edge of the profile. This is also apparent from the GMRT PS at $325 \mathrm{MHz}$ in Figure A1, where the PPA track is clearly delayed with respect to the putative core-component peak. Now, we interpret this displacement of the $\mathrm{SG}$ as indicating that $\mathrm{A} / \mathrm{R}$ plays a strong role in this pulsar's profile form. To fit the RVM to the displaced-PPA track, we use a two-way modeseparation technique (e.g., Gil et al 1993). The RVM fit yields the SG point of the PPA track with good accuracy (see Table A5) and corresponds to the longitude origin in Fig. A1. Moreover, the width of the central core component is measured to be just over $9^{\circ}$, indicates that $\alpha$ is just over $40^{\circ}$.

Changes in PSR B0355+54's profile shape were noted earlier by Morris et al (1980) at $11 \mathrm{~cm}$, where short averages were seen to change slowly from one profile mode to the other over an interval of about 1000 pulses. We see no such mode change in our observation. The pulsar, however, shows sudden "flarings" towards the pulse edges for about $2 \%$ of the total time. Flarings on the leading and the trailing edges of the profile are generally uncorrelated and without any obvious periodicity. The flared profile in Fig. A1 clearly shows what seem to be conal outriders, and its overall form can be well described in terms of the triple $(\mathrm{T})$ or perhaps $\mathrm{M}$ class - therefore we use the hybrid designation $\operatorname{arT} / \mathrm{M}$.

Further the midway point of the peak of the outer components clearly leads the steepest gradi- 
Table A1: Observational Parameters

\begin{tabular}{|c|c|c|c|c|}
\hline $\begin{array}{l}\text { PSR } \\
\text { Bname }\end{array}$ & $\begin{array}{l}\text { Obs'y } \\
\text { Band }\end{array}$ & $\overline{\overline{\text { MJD }}}$ & $\begin{array}{l}\text { length/ } \\
\text { res }\left(^{\circ}\right)\end{array}$ & Fig. \\
\hline B0138+59 & GM:P & 54399 & $1961 / 0.15$ & A1 \\
\hline B $0355+54$ & GM:P & 53780 & $13144 / 1.18$ & A1 \\
\hline B $0450+55$ & GM:P & 53245 & $2671 / 0.54$ & \\
\hline B $0540+23$ & AO:P & 54015 & $2440 / 0.66$ & \\
\hline B0740-28 & GM:P & 53781 & $3649 / 1.10$ & A2 \\
\hline $\mathrm{B} 0809+74$ & GM:P & 54399 & $940 / 0.14$ & 2 \\
\hline B0906-17 & GM:P & 54399 & $2256 / 0.46$ & $\overline{A 2}$ \\
\hline B0919+06 & AO:L & 52854 & $1115 / 0.43$ & 2 \\
\hline B1055-52I & GM:P & 54537 & $16571 / 0.93$ & A3 \\
\hline$B 1112+50$ & GM:P & 54399 & $2002 / 0.11$ & A3 \\
\hline$B 1322+83 m$ & GM:P & 54399 & $2700 / 0.28$ & A3 \\
\hline $\mathrm{B} 1530+27$ & $\mathrm{AO}: \mathrm{P}$ & 53994 & $1032 / 0.33$ & 3 \\
\hline B1540-06 & GM:P & 54399 & $2129 / 0.26$ & A4 \\
\hline B1556-44 & GM:P & 53781 & $3629 / 0.72$ & A4 \\
\hline B1604-00 & AO:L & 53372 & $1605 / 0.22$ & A4 \\
\hline $\mathrm{B} 1612+07$ & $\mathrm{AO}: \mathrm{P}$ & 53378 & $1094 / 0.31$ & 4 \\
\hline B1700-18 & GM:P & 54399 & $1917 / 0.23$ & A5 \\
\hline B1732-07 & GM:P & 54399 & $2015 / 0.42$ & A5 \\
\hline$B 1742-30$ & GM:P & 54399 & $1975 / 0.50$ & A5 \\
\hline$B 1745-12$ & GM:P & 53781 & $2740 / 0.47$ & $\overline{45}$ \\
\hline B1822-09 & GM:P & 54399 & $1962 / 0.24$ & $\mathrm{~A} 6$ \\
\hline$B 1842+14$ & $\mathrm{AO}: \mathrm{P}$ & 53378 & $1600 / 0.46$ & 46 \\
\hline B1851-14 & GM:P & 54399 & $1079 / 0.16$ & $\mathrm{~A} 6$ \\
\hline$B 1900+05$ & AO:L & 54842 & $1045 / 0.39$ & A6 \\
\hline В1907-03 & GM:P & 54399 & $2035 / 0.37$ & A7 \\
\hline $\mathrm{B} 1910+20$ & AO:L & 53372 & $906 / 0.33$ & A7 \\
\hline$B 1913+10$ & $\mathrm{AO}: \mathrm{L}$ & 54538 & $2077 / 0.26$ & 7 \\
\hline $\mathrm{B} 1915+13$ & $\mathrm{AO}: \mathrm{L}$ & 48918 & $4000 / 0.33$ & A7 \\
\hline $\mathrm{B} 1924+16$ & $\mathrm{AO}: \mathrm{L}$ & 54538 & $2522 / 0.26$ & $\mathrm{~A} 8$ \\
\hline B1930+22 & $\mathrm{AO}: \mathrm{L}$ & 54540 & $4151 / 0.64$ & $\mathrm{~A} 8$ \\
\hline B1937-26 & GM:P & 54399 & $1965 / 0.46$ & A8 \\
\hline$B 1944+17$ & $\mathrm{AO}: \mathrm{P}$ & 53966 & $7038 / 0.31$ & A8 \\
\hline$B 1944+22$ & $\mathrm{AO}: \mathrm{P}$ & 55276 & $932 / 0.35$ & A9 \\
\hline $\mathrm{B} 2021+51$ & GM:P & 54399 & $2282 / 0.35$ & A9 \\
\hline B2043-04 & GM:P & 54399 & $1993 / 0.12$ & A9 \\
\hline B2053+36 & $\mathrm{AO}: \mathrm{L}$ & 52837 & $52837 / 0.42$ & A9 \\
\hline $\mathrm{B} 2217+47$ & GM:P & 54399 & $2243 / 0.34$ & A10 \\
\hline $\mathrm{B} 2224+65 \mathrm{~m}$ & GM:P & 54399 & $2101 / 0.27$ & A10 \\
\hline B2327-20 & GM:P & 54399 & $1865 / 0.11$ & A10 \\
\hline
\end{tabular}

Notes: Pulsars with Bnames in normal type appear in L\&M's Table 4; those in italics are denoted as "Partial cones?" in their Table 5 
Table A2: Parameters for Lyne \& Manchester's "Partial Cone" Pulsars.

\begin{tabular}{|c|c|c|c|c|c|c|c|}
\hline $\begin{array}{l}\text { PSR } \\
\text { Bname }\end{array}$ & $\begin{array}{l}\text { PSR } \\
\text { Jname }\end{array}$ & $\begin{array}{l}P_{1} \\
(\mathrm{~s})\end{array}$ & $\begin{array}{l}\log (\tau) \\
(\mathrm{yrs})\end{array}$ & $\begin{array}{l}\log (B) \\
(\mathrm{G})\end{array}$ & $\begin{array}{l}\log (\dot{E}) \\
\left(\operatorname{erg~s}^{-1}\right)\end{array}$ & Fig. & Remarks \\
\hline B0138+59 & J0141+6009 & 1.223 & 7.69 & 11.85 & 30.93 & A1 & - \\
\hline В0254-53 & J0255-5304 & 0.448 & 8.37 & 11.07 & 31.11 & - & $\mathrm{U}$ \\
\hline B0355+54 & J0358+5413 & 0.156 & 5.75 & 11.92 & 34.66 & A1 & $\mathrm{T}$ \\
\hline B0450+55 & J0454+5543 & 0.341 & 6.36 & 11.96 & 33.38 & A1 & $\mathrm{T}$ \\
\hline B0540+23 & $\mathrm{J} 0543+2329$ & 0.246 & 5.40 & 12.29 & 34.61 & A1 & $\mathrm{T}$ \\
\hline$B 0643+80$ & J0653+8051 & 1.214 & 6.71 & 12.34 & 31.92 & - & $\mathrm{U}$ \\
\hline B0740-28 & J0742-2822 & 0.167 & 5.20 & 12.23 & 35.16 & A2 & $\mathrm{T}$ \\
\hline B0809+74 & J0814+7429 & 1.292 & 8.09 & 11.67 & 30.49 & $\mathrm{~A} 2$ & $\mathrm{~T}$ \\
\hline B0906-17 & J0908-1739 & 0.402 & 6.98 & 11.72 & 32.61 & $\mathrm{~A} 2$ & $\mathrm{U}$ \\
\hline B0919+06 & J0922+0638 & 0.431 & 5.70 & 12.39 & 33.83 & $\mathrm{~A} 2$ & $\mathrm{~T}$ \\
\hline B1055-52I & J1057-5226 & 0.198 & 5.73 & 12.04 & 34.48 & A3 & $\mathrm{T}$ \\
\hline$B 1112+50$ & $\mathrm{~J} 1115+5030$ & 1.656 & 7.02 & 12.31 & 31.34 & A3 & $\mathrm{U}$ \\
\hline B1221-63 & J1224-6407 & 0.216 & 5.84 & 12.02 & 34.28 & - & $\mathrm{U}$ \\
\hline B1240-64 & J1243-6423 & 0.388 & 6.14 & 12.13 & 33.48 & - & $\mathrm{U}$ \\
\hline$B 1322+83 m$ & $\mathrm{~J} 1321+8323$ & 0.670 & 7.27 & 11.80 & 31.87 & A3 & - \\
\hline B1356-60 & J1359-6038 & 0.128 & 5.50 & 11.96 & 35.08 & - & $\mathrm{U}$ \\
\hline B1426-66 & J1430-6623 & 0.785 & 6.65 & 12.17 & 32.36 & - & $\mathrm{U}$ \\
\hline B1449-64 & J1453-6413 & 0.179 & 6.02 & 11.85 & 34.28 & - & $\mathrm{U}$ \\
\hline $\mathrm{B} 1530+27$ & $\mathrm{~J} 1532+2745$ & 1.125 & 7.36 & 11.98 & 31.33 & A3 & $\mathrm{U}$ \\
\hline B1530-53 & J1534-5334 & 1.369 & 7.18 & 12.15 & 31.34 & - & - \\
\hline B1540-06 & J1543-0620 & 0.709 & 7.11 & 11.90 & 31.99 & A4 & $\mathrm{U}$ \\
\hline B1556-44 & J1559-4438 & 0.257 & 6.60 & 11.71 & 33.38 & A4 & $\mathrm{T}$ \\
\hline B1604-00 & J1607-0032 & 0.422 & 7.34 & 11.56 & 32.21 & A4 & $\mathrm{T}$ \\
\hline B1612+07 & $\mathrm{J} 1614+0737$ & 1.207 & 6.91 & 12.23 & 31.72 & A4 & $\mathrm{U}$ \\
\hline B1641-45 & J1644-4559 & 0.455 & 5.56 & 12.49 & 33.92 & - & $\mathrm{U}$ \\
\hline B1648-42 & J1651-4246 & 0.844 & 6.44 & 12.31 & 32.51 & - & $\mathrm{U}$ \\
\hline B1700-18 & J1703-1846 & 0.804 & 6.87 & 12.08 & 32.12 & A5 & $\mathrm{T}$ \\
\hline B1732-07 & J1735-0724 & 0.419 & 6.74 & 11.86 & 32.81 & A5 & $\mathrm{U}$ \\
\hline B1742-30 & J1745-3040 & 0.367 & 5.74 & 12.30 & 33.93 & A5 & $\mathrm{U}$ \\
\hline$B 1745-12$ & J1748-1300 & 0.394 & 6.71 & 11.85 & 32.89 & A5 & $\mathrm{T}$ \\
\hline B1756-22 & J1759-2205 & 0.461 & 5.83 & 12.36 & 33.64 & - & $\mathrm{U}$ \\
\hline B1822-09 & J1825-0935 & 0.769 & 5.37 & 12.81 & 33.66 & A6 & - \\
\hline$B 1842+14$ & $\mathrm{~J} 1844+1454$ & 0.375 & 6.50 & 11.93 & 33.15 & $\overline{\mathrm{A} 6}$ & $\mathrm{U}$ \\
\hline B1851-14 & J1854-1421 & 1.147 & 6.64 & 12.34 & 32.04 & A6 & U \\
\hline (B1859+07) & $\mathrm{J} 1901+0716$ & 0.644 & 6.65 & 12.09 & 32.53 & - & \\
\hline$B 1900+05$ & $\mathrm{~J} 1902+0556$ & 0.747 & 5.96 & 12.50 & 33.08 & A6 & U \\
\hline В1907-03 & J1910-0309 & 0.505 & 6.56 & 12.03 & 32.83 & A7 & U \\
\hline $\mathrm{B} 1910+20$ & $\mathrm{~J} 1912+2104$ & 2.233 & 6.54 & 12.68 & 31.56 & A7 & $\mathrm{T}$ \\
\hline$B 1913+10$ & $\mathrm{~J} 1915+1009$ & 0.405 & 5.62 & 12.40 & 33.96 & A7 & U \\
\hline B1915+13 & $\mathrm{J} 1917+1353$ & 0.195 & 5.63 & 12.08 & 34.59 & A7 & $\mathrm{T}$ \\
\hline B1924+16 & $\mathrm{J} 1926+1648$ & 0.580 & 5.71 & 12.52 & 33.56 & A8 & $\mathrm{T}$ \\
\hline B1930+22 & $\mathrm{J} 1932+2220$ & 0.144 & 4.60 & 12.47 & 35.88 & A8 & $\mathrm{T}$ \\
\hline B1937-26 & J1941-2602 & 0.403 & 6.82 & 11.80 & 32.76 & A8 & $\mathrm{T}$ \\
\hline$B 1944+17$ & $\mathrm{~J} 1946+1805$ & 0.441 & 8.46 & 11.02 & 31.041 & $\mathrm{A8}$ & $\mathrm{U}$ \\
\hline$B 1944+22$ & $\mathrm{~J} 1946+2244$ & 1.334 & 7.38 & 12.04 & 31.18 & A9 & $\mathrm{U}$ \\
\hline $\mathrm{B} 2021+51$ & $\mathrm{~J} 2022+5154$ & 0.529 & 6.44 & 12.11 & 32.91 & A9 & $\mathrm{T}$ \\
\hline B2043-04 & J2046-0421 & 1.547 & 7.22 & 12.18 & 31.20 & A9 & - \\
\hline $\mathrm{B} 2053+36$ & $\mathrm{~J} 2055+3630$ & 0.222 & 6.989 & 11.46 & 33.13 & A9 & $\mathrm{U}$ \\
\hline $\mathrm{B} 2217+47$ & $\mathrm{~J} 2219+4754$ & 0.538 & 6.49 & 12.09 & 32.84 & A10 & - \\
\hline B2224+65m & $\mathrm{J} 2225+6535$ & 0.683 & 6.05 & 12.41 & 33.08 & A10 & $\mathrm{U}$ \\
\hline B2327-20 & J2330-2005 & 1.644 & 6.75 & 12.45 & 31.61 & A10 & - \\
\hline
\end{tabular}


Table A3: Emission-Beam Geometry of "Partial Cone" Pulsars

\begin{tabular}{|c|c|c|c|c|c|c|c|c|c|c|c|c|}
\hline PSR & Class & $\alpha$ & $\begin{array}{c}|\Delta \chi / \Delta \varphi|_{o} \\
\left(\% /{ }^{\circ}\right)\end{array}$ & $\beta$ & $\Delta \Psi$ & $\begin{array}{c}\text { Inner } \\
\rho \\
\end{array}$ & $\beta / \rho$ & $\Delta \Psi$ & $\begin{array}{c}\text { Outer } \\
\rho\end{array}$ & $\beta / \rho$ & $\begin{array}{l}\text { Inner } \\
\text { Inner }\end{array}$ & $\begin{array}{c}(\mathrm{km})_{-} \\
\text {Outer }\end{array}$ \\
\hline B0138+59 & $\mathrm{M} / \mathrm{cQ} ?$ & 20 & -11.2 & 1.7 & $\sim-$ & - & - & 27 & 5.1 & 0.34 & - & 211 \\
\hline B0254-53 & D? & 55 & $-8 ?$ & 5.9 & 7 & 6.6 & 0.89 & - & - & - & $129 ?$ & - \\
\hline B0355+54 & $\operatorname{arT} / \mathrm{M}$ & 42 & -9.2 & 4.2 & - & - & - & 40 & 14.6 & 0.29 & - & 221 \\
\hline B $0450+55$ & $\operatorname{arT}$ & 32 & -8.5 & 3.5 & - & - & - & 34 & 10.0 & 0.35 & - & 226 \\
\hline B $0540+23$ & $\mathrm{D} / \mathrm{T} ?$ & $30 ?$ & -3.4 & 8.5 & - & - & - & $29 ?$ & 11.7 & 0.72 & - & 224 \\
\hline$B 0643+80$ & $\mathrm{Sd} / \mathrm{D} ?$ & 22 & +6 & 3.6 & 9 & 4.0 & 0.89 & - & - & - & 130 & - \\
\hline B0740-28 & arM? & $90 ?$ & -5.5 & 10.5 & $7 ?$ & 11.0 & 0.95 & $18 ?$ & 13.8 & 0.76 & 135 & 211 \\
\hline $\mathrm{B} 0809+74^{b}$ & $\mathrm{Sd}$ & 8.8 & -1.8 & 4.9 & - & - & - & 17.0 & 5.1 & 0.95 & - & 227 \\
\hline B0906-17 & $\operatorname{arT}$ & 31 & $-6 ?$ & 4.9 & $17 ?$ & 6.8 & 0.73 & - & - & - & 124 & - \\
\hline $\mathrm{B} 0919+06^{c}$ & $\mathrm{~T}$ & 53 & +9 & 5.1 & 10 & 6.5 & 0.78 & - & - & - & 122 & - \\
\hline $\mathrm{B} 1055-52 \mathrm{i}^{d}$ & M? & 22 & +9.1 & 2.4 & - & - & - & $65 ?$ & 13.1 & 0.18 & - & 224 \\
\hline$B 1112+50$ & St? & 30 & +10.1 & 2.8 & 7 & 3.4 & 0.84 & - & - & - & 126 & - \\
\hline B1221-63 & $\mathrm{T} ?$ & 61 & +7 & 7.2 & 13 & 9.3 & 0.77 & - & - & - & 125 & - \\
\hline B1240-64 & $\mathrm{St}$ & 69 & +15 & 3.6 & $13 ?$ & 7.1 & 0.50 & - & - & - & 131 & - \\
\hline$B 1322+83 m$ & Sd? & 14 & +2.8 & 5.1 & $12 ?$ & 5.4 & 0.95 & - & - & - & 130 & - \\
\hline B1356-60 & St & 79 & $+3^{a}$ & 19.1 & - & - & - & - & - & - & - & - \\
\hline B1426-66 & $\mathrm{T}$ & 54 & $-50^{a}$ & 0.9 & 12 & 5.0 & 0.19 & - & - & - & 131 & - \\
\hline B1449-64 & $\mathrm{St}$ & 43 & $+7^{a}$ & 5.6 & $25 ?$ & 10.5 & 0.53 & - & - & - & 132 & - \\
\hline $\mathrm{B} 1530+27 \mathrm{~m}$ & $\mathrm{Sd} / \mathrm{D} ?$ & 30 & +5.8 & 4.9 & - & - & - & 9 & 5.5 & 0.90 & - & 225 \\
\hline B1530-53 & $\mathrm{D} ?$ & 22 & $-18^{a}$ & 1.2 & $19 ?$ & 3.8 & 0.31 & - & - & - & 128 & - \\
\hline B1540-06 & $\mathrm{Sd}$ & 59 & $-14 ?$ & 3.5 & $9 ?$ & 5.3 & 0.67 & - & - & - & 131 & - \\
\hline B1556-44 & $\mathrm{St} / \mathrm{T}$ & 32 & $-9^{a}$ & 3.4 & 28 & 8.6 & 0.40 & - & - & - & 125 & - \\
\hline B1604-00 & $\mathrm{cT}$ & 50 & $-8 ?$ & 5.5 & 9.8 & 6.7 & 0.82 & - & - & - & 128 & - \\
\hline $\mathrm{B} 1612+07$ & $\mathrm{Sd}$ & 25 & -4.6 & 5.2 & - & - & - & 4.5 & 5.3 & 0.98 & - & 224 \\
\hline B1641-45 & $\mathrm{St}$ & 33 & $\infty$ & 0.0 & 24 & 6.5 & 0.0 & - & - & - & 128 & - \\
\hline B1648-42 & $\mathrm{D} / \mathrm{cT}$ & 6.5 & $-4^{a}$ & 1.6 & - & - & - & 100 & 6.3 & 0.26 & - & 226 \\
\hline B1700-18 & $\mathrm{Sd}$ & 44 & -8.2 & 4.7 & - & - & - & 12 & 6.4 & 0.74 & - & 221 \\
\hline B1732-07 & $\mathrm{T} ?$ & 54 & $\infty$ & 0.0 & 17 & 6.8 & 0.0 & - & - & - & 131 & - \\
\hline B1742-30 & M & 24 & -3.6 & 6.4 & $15 ?$ & 7.3 & 0.89 & 32 & 9.7 & 0.67 & 129 & 228 \\
\hline B1745-12 & $\mathrm{T} / \mathrm{cQ} ?$ & 75 & -11.7 & 4.9 & - & - & - & $16 ?$ & 9.2 & 0.53 & - & 222 \\
\hline B1756-22 & $\mathrm{St} / \mathrm{T} ?$ & $\sim 90 ?$ & $\infty$ & 0.0 & $12 ?$ & 6.2 & 0.0 & - & - & - & 118 & - \\
\hline $\mathrm{B} 1822-09 \mathrm{~m}^{e}$ & $\mathrm{~T}$ & 86 & $\infty$ & 0.0 & 10.0 & 5.0 & 0.0 & - & - & - & 128 & - \\
\hline$B 1842+14$ & St? & 63 & +12 & 4.2 & 12 & 6.9 & 0.62 & - & - & - & 119 & - \\
\hline B1851-14 & Sd? & $34 ?$ & -7.8 & 4.1 & - & - & - & $12 ?$ & 5.4 & 0.76 & - & 224 \\
\hline$(\mathrm{B} 1859+07)^{c}$ & $\mathrm{~T} / \mathrm{M} ?$ & 30 & +6 & 4.8 & - & - & - & 20 & 7.1 & 0.67 & - & 219 \\
\hline$B 1900+05$ & St? & $59 ?$ & $\infty$ & 0.0 & $12 ?$ & 5.1 & 0.0 & - & - & - & 132 & - \\
\hline В1907-03 & $\mathrm{St} / \mathrm{T}$ & 44 & $\infty$ & 0.0 & 18 & 6.2 & 0.0 & - & - & - & 129 & - \\
\hline $\mathrm{B} 1910+20$ & $\mathrm{cQ} / \mathrm{M}$ & 32 & +30 & 1.0 & $\sim 10.5$ & 3.0 & 0.34 & 14 & 3.9 & 0.26 & 133 & 225 \\
\hline$B 1913+10$ & St? & 64 & $?$ & - & - & - & - & - & - & - & - & - \\
\hline B1915+13 & arSt & 68 & -9.8 & 5.4 & - & - & - & - & - & - & - & - \\
\hline B1924+16 & $\operatorname{arSt}$ & 34 & +5.2 & 6.2 & - & - & - & - & - & - & - & - \\
\hline B1930+22 & arSt? & - & +8.6 & - & - & - & - & - & - & - & - & - \\
\hline B1937-26 & $\mathrm{T} ?$ & 42 & -4.5 & 8.5 & - & - & - & 9 & 9.1 & 0.94 & - & 222 \\
\hline$B 1944+17 f$ & $\mathrm{cT} / \mathrm{cQ}$ & 5 & +0.8 & 6.3 & $\sim 95 ?$ & 8.7 & 0.72 & 30 & 6.6 & 0.95 & 222 & 126 \\
\hline$B 1944+22$ & $\mathrm{Sd} / \mathrm{D} ?$ & 40 & $-12 ?$ & 3.1 & $7 ?$ & 3.8 & 0.80 & - & - & - & 132 & - \\
\hline B2021+51 & Sd? & 30 & +3.9 & 7.3 & - & - & - & 10.3 & 7.8 & 0.93 & - & 216 \\
\hline B2043-04 & $\mathrm{Sd} / \mathrm{D}$ & 73 & +27.1 & 2.0 & $6 ?$ & 3.5 & 0.57 & - & - & - & 128 & - \\
\hline B2053+36 & St & 34 & $\infty$ & -20 & - & - & - & - & - & - & - & - \\
\hline $\mathrm{B} 2217+47$ & St & 42 & +8.5 & 4.5 & 12.0 & 6.1 & .73 & - & - & - & 135 & - \\
\hline $\mathrm{B} 2224+65 \mathrm{~m}$ & St? & 27 & -3.6 & 4.9 & - & - & - & - & - & - & - & - \\
\hline B2327-20 & $\mathrm{T} ?$ & 66 & +43 & 1.2 & 7.0 & 3.4 & 0.35 & - & - & - & 128 & - \\
\hline
\end{tabular}


Table A4: Table of A/R height estimates

\begin{tabular}{lllllll}
\hline \hline $\begin{array}{l}\text { PSR } \\
\text { Bname }\end{array}$ & $\begin{array}{l}P_{1} \\
(\mathbf{s e c})\end{array}$ & $\begin{array}{l}\text { Left width } \\
\left({ }^{\circ}\right)\end{array}$ & $\begin{array}{l}\text { Right Width } \\
\left({ }^{\circ}\right)\end{array}$ & $\begin{array}{l}\sigma_{\phi_{\circ}} \\
\left({ }^{\circ}\right)\end{array}$ & $\begin{array}{l}\text { Shift/ } \\
\left({ }^{\circ}\right)\end{array}$ & $\begin{array}{l}\text { Height } \\
(\mathbf{k m})\end{array}$ \\
\hline $\mathrm{B} 0355+54$ & 0.156 & $-39.7 \pm 0.5$ & $9.3 \pm 0.4$ & 0.5 & $15.2 \pm 0.6$ & $494 \pm 19$ \\
$\mathrm{~B} 0450+55$ & 0.340 & $-18.5 \pm 0.2$ & $10.8 \pm 0.2$ & 0.1 & $3.9 \pm 0.2$ & $275 \pm 12$ \\
$\mathrm{~B} 1700-18^{*}$ & 0.804 & $-6.6 \pm 0.1$ & $3.3 \pm 0.1$ & 1.2 & $1.6 \pm 1.2$ & $279 \pm 201$ \\
$\mathrm{~B} 1732-07^{*}$ & 0.419 & $-10.0 \pm 0.2$ & $5.3 \pm 0.2$ & 0.1 & $2.3 \pm 0.2$ & $205 \pm 15$ \\
$\mathrm{~B} 1742-30$ & 0.367 & $-16.2 \pm 0.1$ & $13.9 \pm 0.5$ & 0.1 & $1.1 \pm 0.3$ & $87 \pm 20$ \\
$\mathrm{~B} 1745-12$ & 0.394 & $-12.0 \pm 0.5$ & $6.8 \pm 0.5$ & 0.8 & $2.6 \pm 0.8$ & $215 \pm 71$ \\
$\mathrm{~B} 1910+20$ & 2.232 & $-6.4 \pm 0.1$ & $5.4 \pm 0.1$ & 0.1 & $0.5 \pm 0.1$ & $228 \pm 56$ \\
$\mathrm{~B} 1924+16$ & 0.579 & $-17.0 \pm 0.3$ & $8.6 \pm 0.3$ & 0.7 & $4.2 \pm 0.7$ & $506 \pm 88$ \\
$\mathrm{~B} 1930+22$ & 0.144 & $-14.5 \pm 0.3$ & $-2.2 \pm 0.3$ & 1.5 & $8.3 \pm 1.5$ & $250 \pm 45$ \\
$\mathrm{~B} 1937-26$ & 0.402 & $-3.9 \pm 0.2$ & $2.2 \pm 0.2$ & 1.5 & $0.8 \pm 1.5$ & $71 \pm 126$ \\
$\mathrm{~B} 2021+51$ & 0.529 & $-18.3 \pm 0.2$ & $16.2 \pm 0.2$ & 0.6 & $1.0 \pm 0.6$ & $113 \pm 67$ \\
$\mathrm{~B} 2043-04$ & 1.546 & $-3.5 \pm 0.1$ & $4.1 \pm 0.1$ & 1.6 & $-0.2 \pm 1.6$ & $-96 \pm 515$ \\
$\mathrm{~B} 2327-20$ & 1.643 & $-2.6 \pm 0.1$ & $3.0 \pm 0.1$ & 0.4 & $-0.1 \pm 0.4$ & $-68 \pm 139$ \\
\hline
\end{tabular}

Notes: The table gives the pulsar name, period, measured outer conal left and right widths and the shift w.r.t the SG point ( ${ }^{*}$ or core component) which is taken as the longitude origin. The estimated $\mathrm{A} / \mathrm{R}$ heights are given in the last column.

Table A5: Table of RVM fitting results

\begin{tabular}{llll}
\hline \hline PSR & $\begin{array}{l}\sigma_{\chi_{\circ}} \\
\left({ }^{\circ}\right)\end{array}$ & $\begin{array}{l}\sigma_{\phi_{\circ}} \\
\left({ }^{\circ}\right)\end{array}$ & $\begin{array}{l}\mathbf{R} \\
\left({ }^{\circ} /{ }^{\circ}\right)\end{array}$ \\
\hline $\mathrm{B} 0138+59$ & 3 & 0.1 & $-11.2 \pm 0.1$ \\
$\mathrm{~B} 0355+54$ & 2 & 0.5 & $-9.2 \pm 0.1$ \\
$\mathrm{~B} 0450+55$ & 12 & 0.1 & $-8.5 \pm 0.1$ \\
$\mathrm{~B} 0540+23$ & 15 & 1.5 & $-3.4 \pm 0.2$ \\
$\mathrm{~B} 0740-28$ & 5 & 1.3 & $-5.5 \pm 0.4$ \\
$\mathrm{~B} 0809+74$ & 7 & 2 & $-3.4 \pm 0.5$ \\
$\mathrm{~B} 0906-17$ & 9 & 2 & $-2.3 \pm 0.4$ \\
$\mathrm{~B} 0919+06$ & 6 & 1 & $11.8 \pm 1$ \\
$\mathrm{~B} 1112+50$ & 7 & 0.7 & $10.1 \pm 1$ \\
$\mathrm{~B} 1322+83$ & 5 & 1 & $2.8 \pm 0.1$ \\
$\mathrm{~B} 1530+27$ & 7 & 0.6 & $5.8 \pm 1$ \\
$\mathrm{~B} 1700-18$ & 9 & 1.2 & $-8.2 \pm 1$ \\
$\mathrm{~B} 1742-30$ & 15 & $? ? ?$ & $? ? ?$ \\
$\mathrm{~B} 1745-12$ & 10 & 0.8 & $-11.7 \pm 0.5$ \\
$\mathrm{~B} 1910+20$ & 4 & 0.1 & $30 \pm 1$ \\
$\mathrm{~B} 1915+13$ & 4 & 0.5 & $-9.8 \pm 0.4$ \\
$\mathrm{~B} 1924+16$ & 8 & 0.7 & $5.2 \pm 0.4$ \\
$\mathrm{~B} 1930+22$ & 9 & 1.5 & $8.6 \pm 1$ \\
$\mathrm{~B} 1937-26$ & 5 & 1.5 & $-4.5 \pm 0.5$ \\
$\mathrm{~B} 1944+17$ & 3 & 0.9 & $0.8 \pm 0.1$ \\
$\mathrm{~B} 2021+51$ & 5 & 0.6 & $3.9 \pm 0.4$ \\
$\mathrm{~B} 2043-04$ & 8 & 1.6 & $27.1 \pm 3$ \\
$\mathrm{~B} 2224+65$ & 4 & 0.2 & $-3.6 \pm 0.3$ \\
$\mathrm{~B} 2327-20$ & 2 & 0.1 & $43 \pm 1.5$ \\
\hline & & &
\end{tabular}



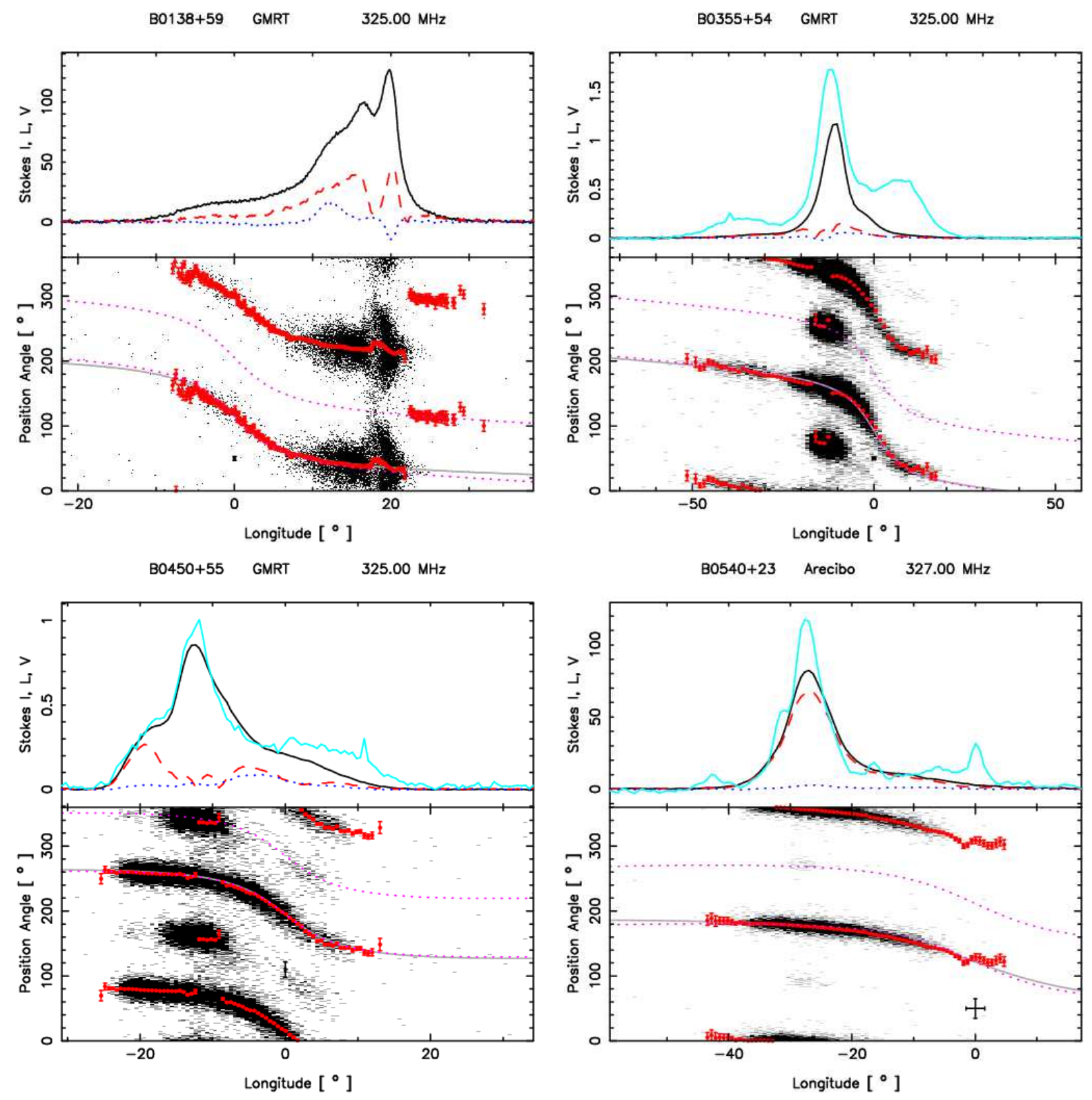

Fig. A1. - PPA histograms and "flared"-emission profiles for "partial cone" pulsars B0138+59, B0355+54, B0450+55 and B0540+23, where the instrument and band is indicated above each plot. The respective upper panels give the total power (black), total linear (red) and circular polarization LH-RH (blue). The cyan curve (if plotted) correspond to the computed flared profile (see text for details). The lower panels give the polarization-angle (hereafter PPA) density, plotted twice for clarity. In cases where PPA fits were possible, a solid grey curve depicts the results of Table A5, the longitude origin is taken at the corresponding steepest gradient point (hereafter SG), and error bars show its uncertainty; whereas the two dotted (magenta) curves indicate the primary- and secondary-mode (hereafter PPM and SPM) PPA traverses corresponding to the geometric models in Table A3. Otherwise, when no RVM fitting information was available, the zero longitude was usually chosen as the peak of the profile (unless mentioned otherwise in notes on each pulsar in this Appendix). 

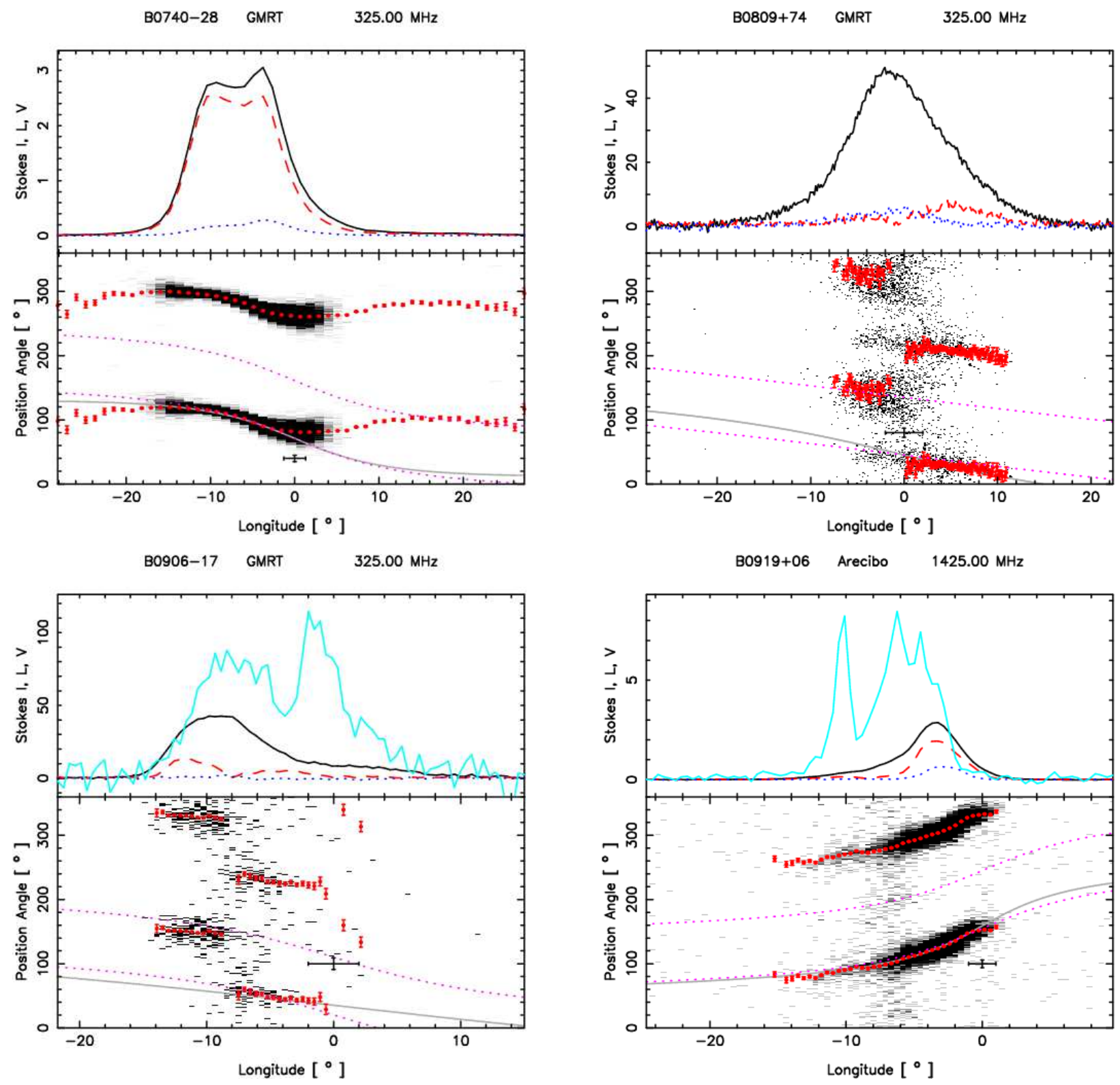

Fig. A2.- PPA histograms and "flared"-emission profiles as in Fig. A1 for pulsars B0740-28, B0809+74, B0906-17 and B0919+06. 

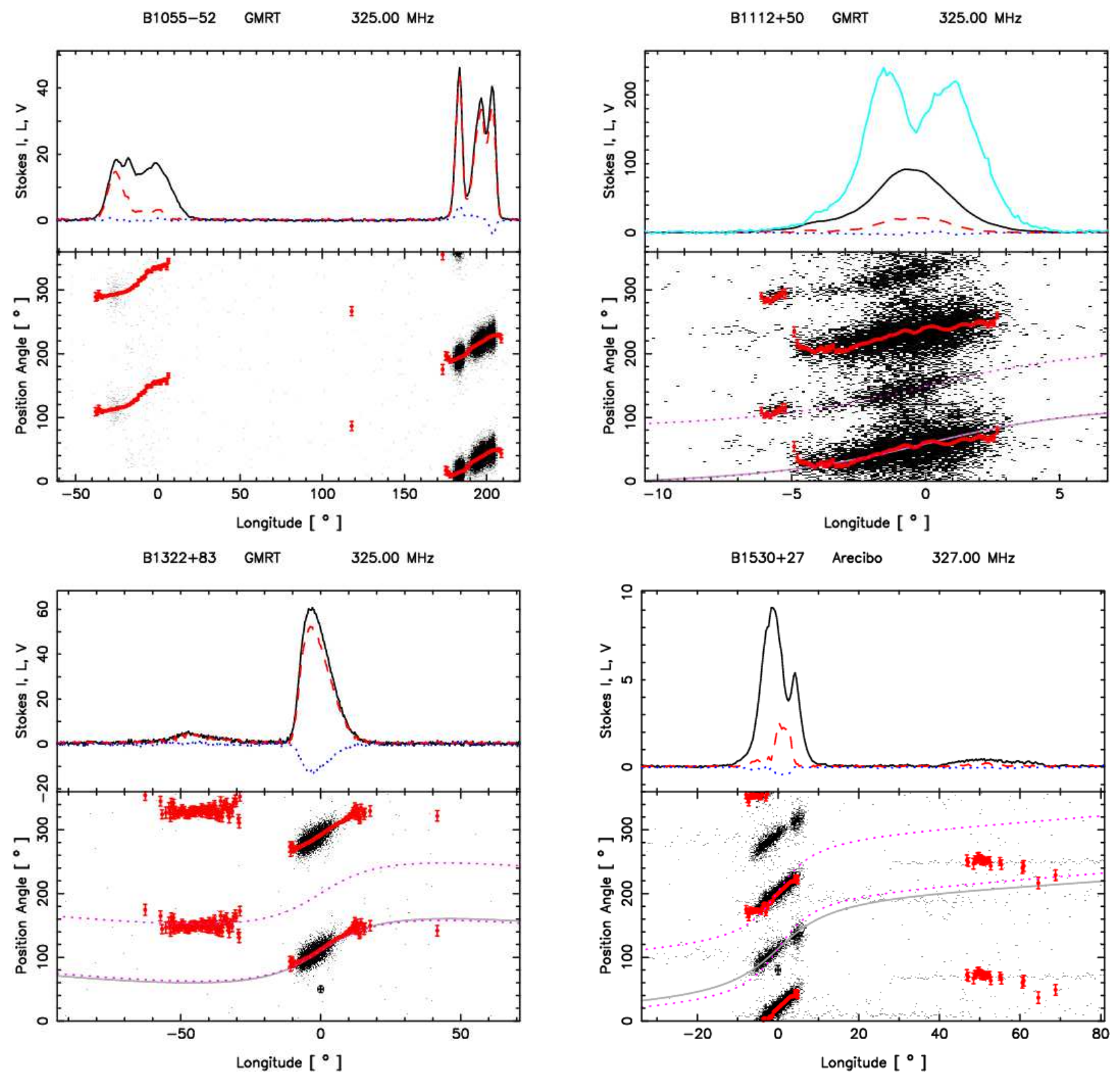

Fig. A3.- PPA histograms and "flared"-emission profiles as in Fig. A1 for pulsars B1055-52, B1112+50, $\mathrm{B} 1322+83$ and $\mathrm{B} 1530+27$. 

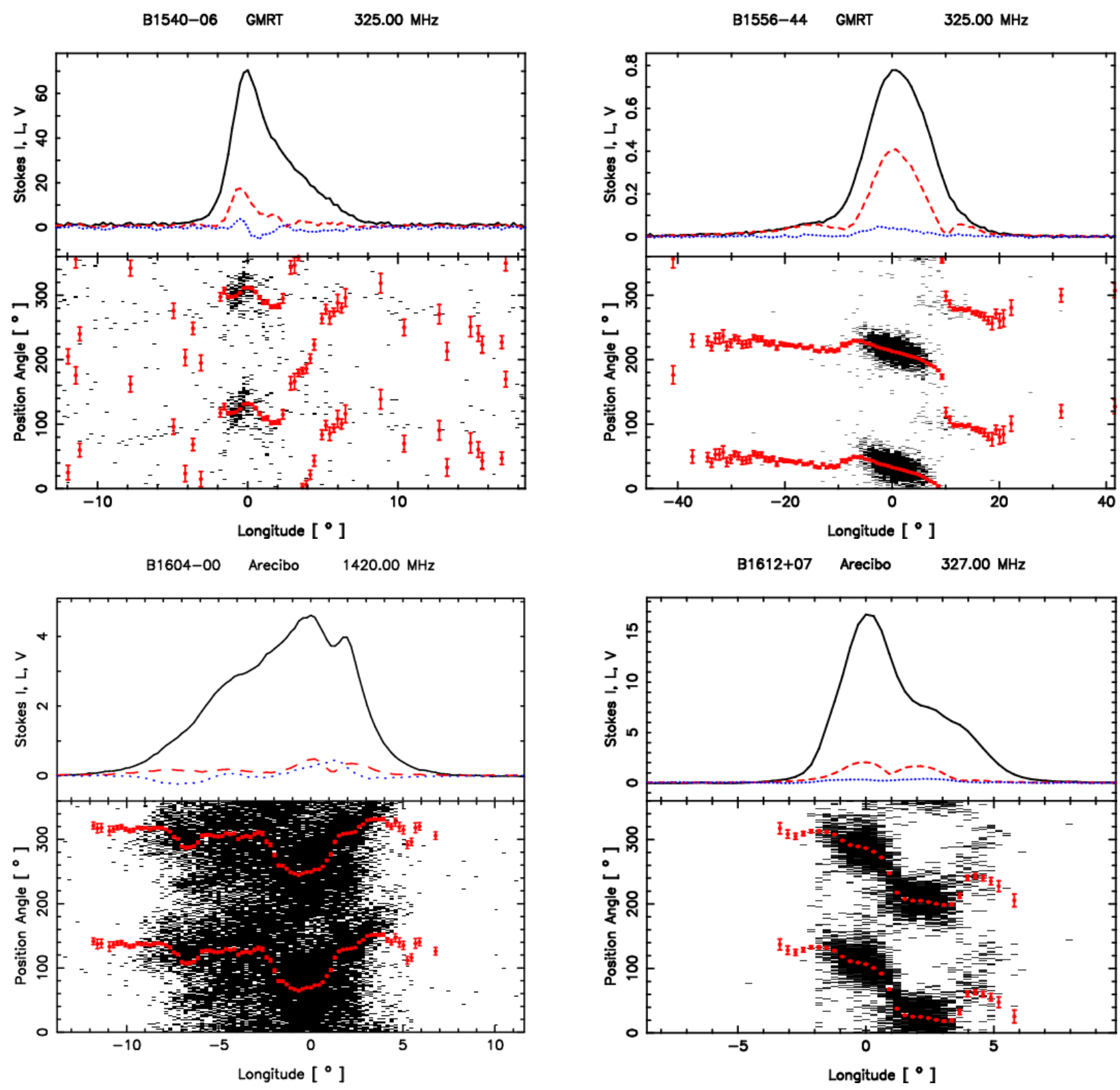

Fig. A4.- PPA histograms and "flared"-emission profiles as in Fig. A1 for pulsars B1540-06, B1556-44, B1604-00 and B1612+07. 

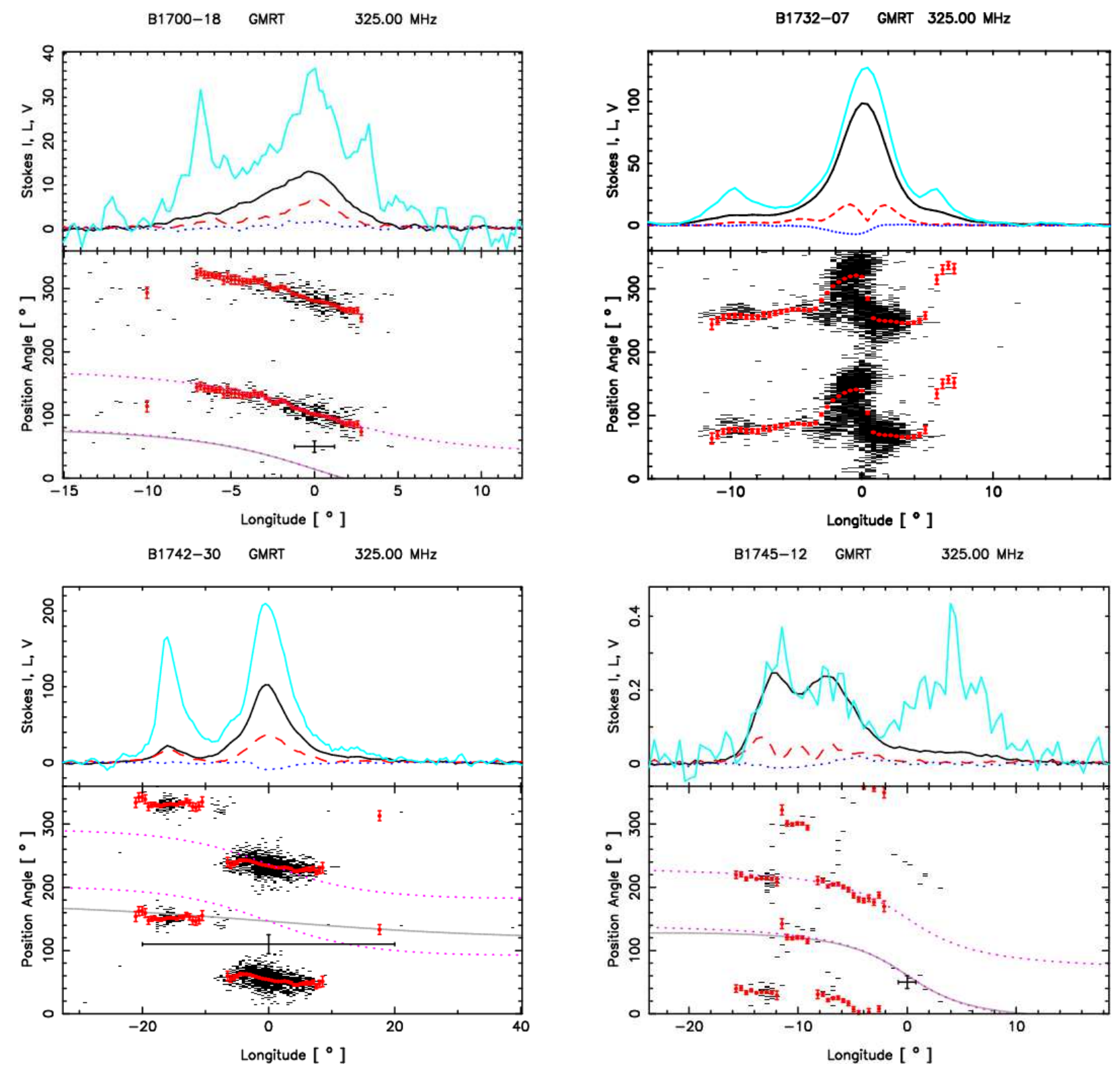

Fig. A5.- PPA histograms and "flared"-emission profiles as in Fig. A1 for pulsars B1700-18, B1732-07, B1742-30 and B1745-12. 

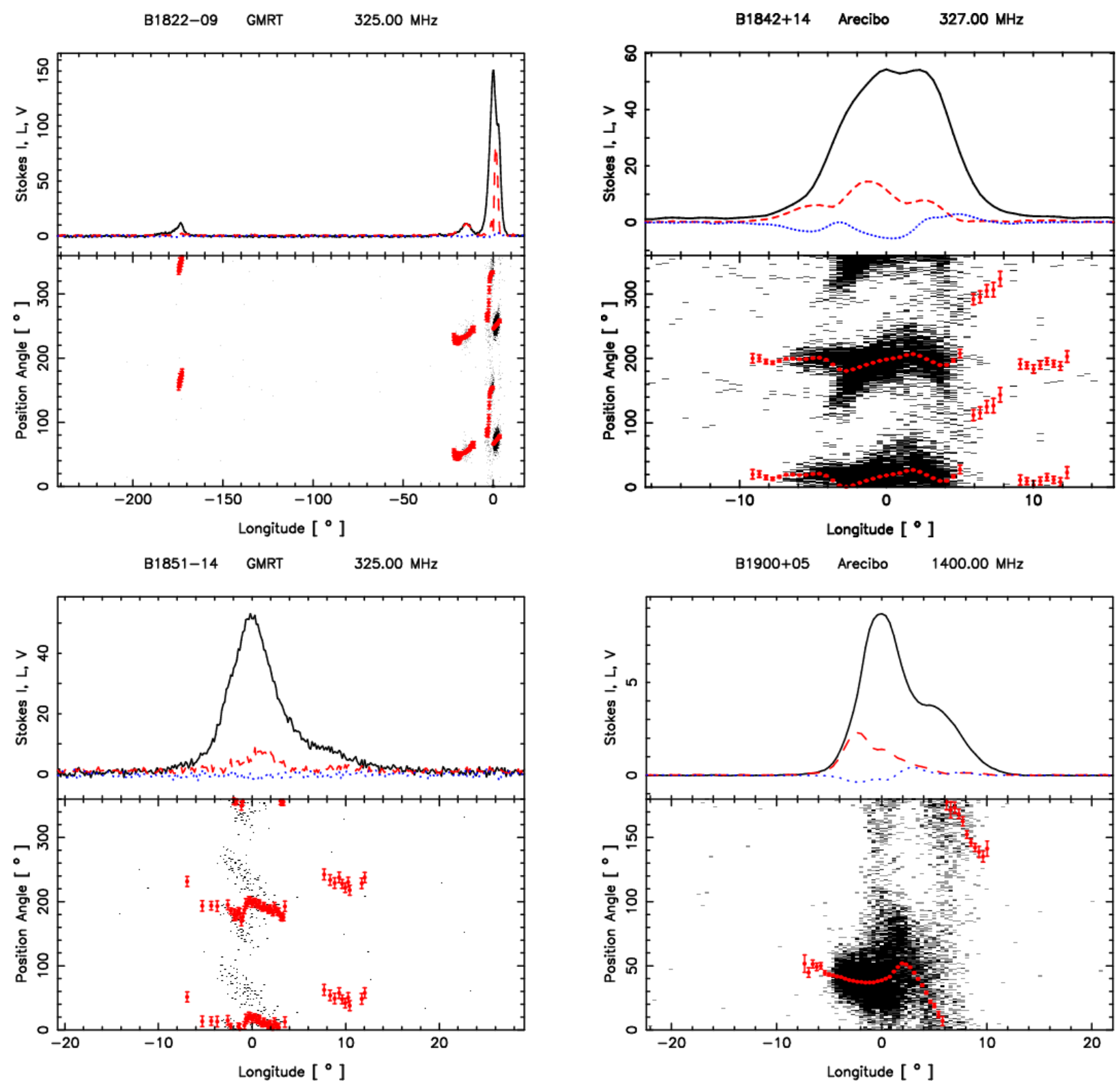

Fig. A6.- PPA histograms and "flared"-emission profiles as in Fig. A1 for pulsars B1822-09, B1842+14, B1851-14 and B1900+05. 

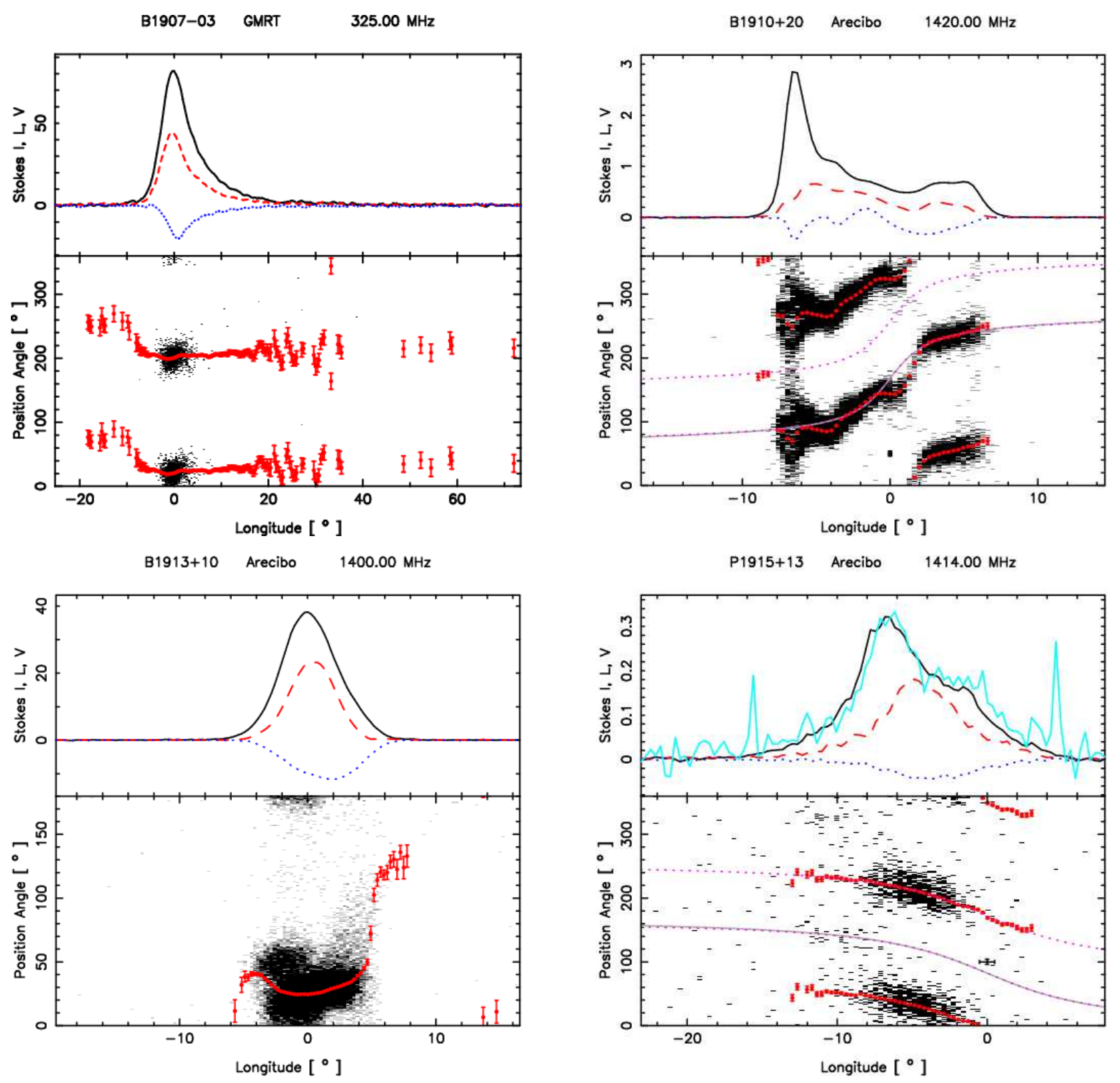

Fig. A7. - PPA histograms and "flared"-emission profiles as in Fig. A1 for pulsars B1907-03, B1910+20, $\mathrm{B} 1913+10$ and B1915+13. 

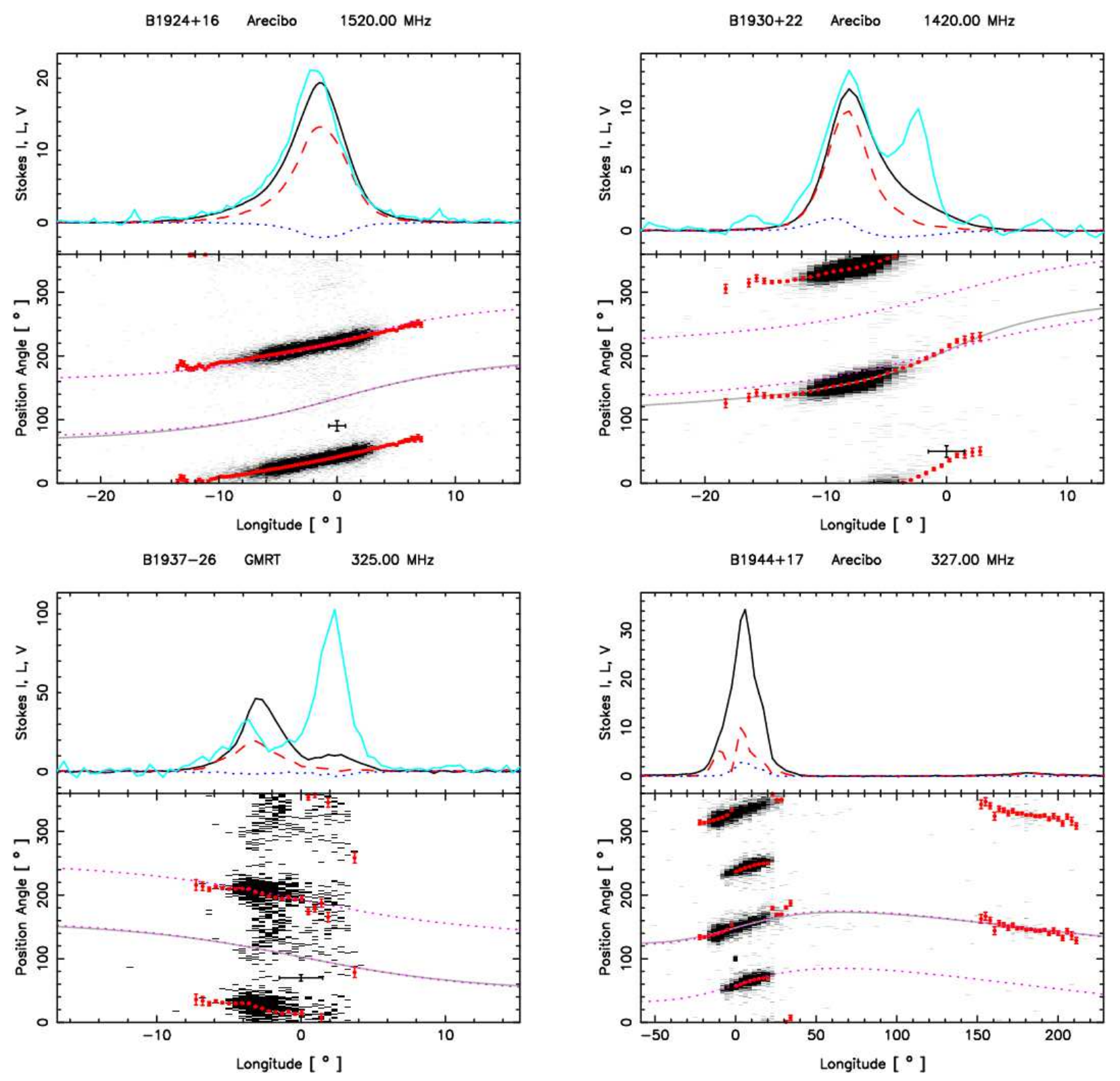

Fig. A8. - PPA histograms and "flared"-emission profiles as in Fig. A1 for pulsars B1924+16, B1930+22, B1937-26 and B1944+17. 

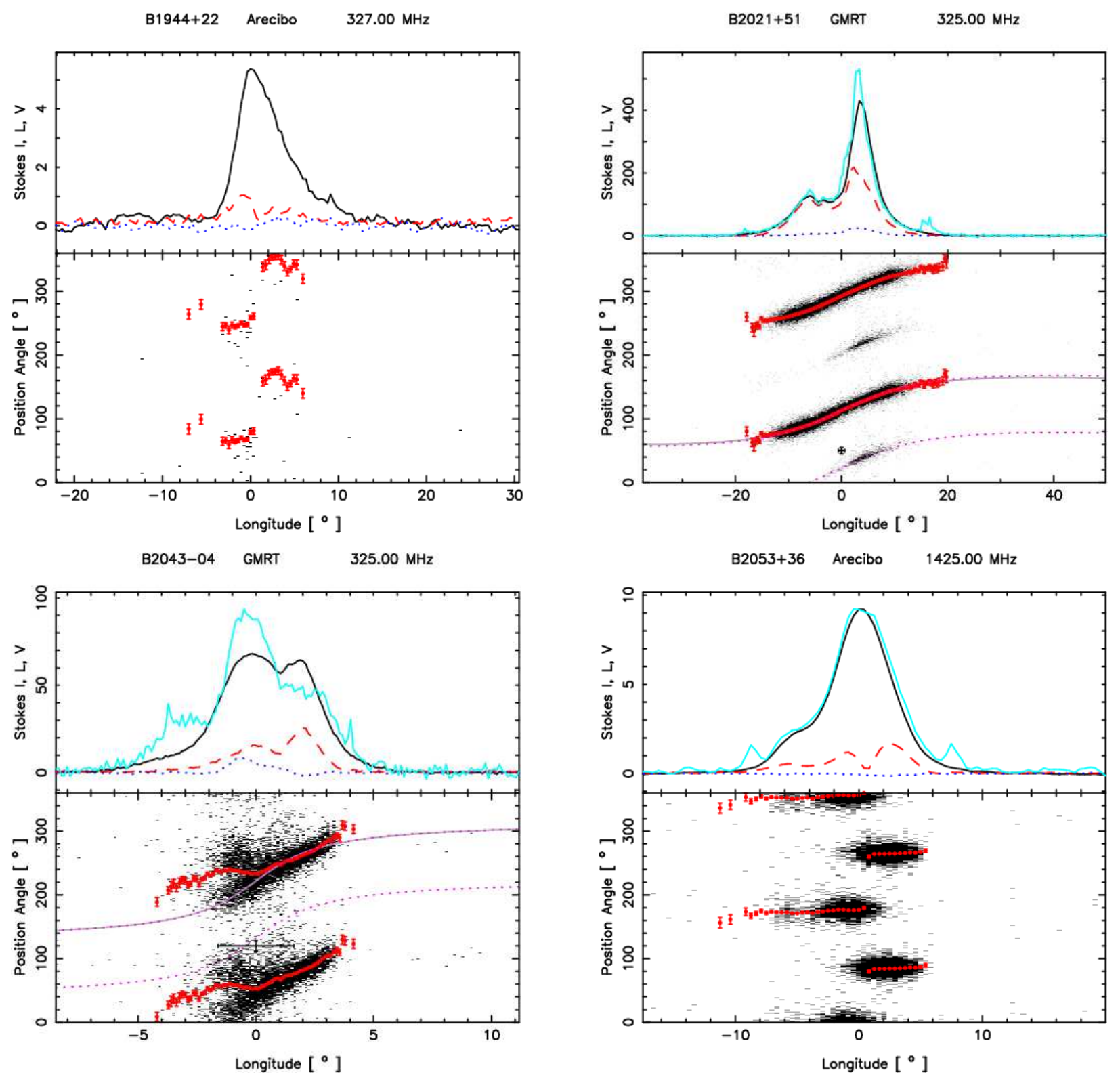

Fig. A9.- PPA histograms and "flared"-emission profiles as in Fig. A1 for pulsars B1944+22, B2021+51, B2043-04 and B2053+36. 

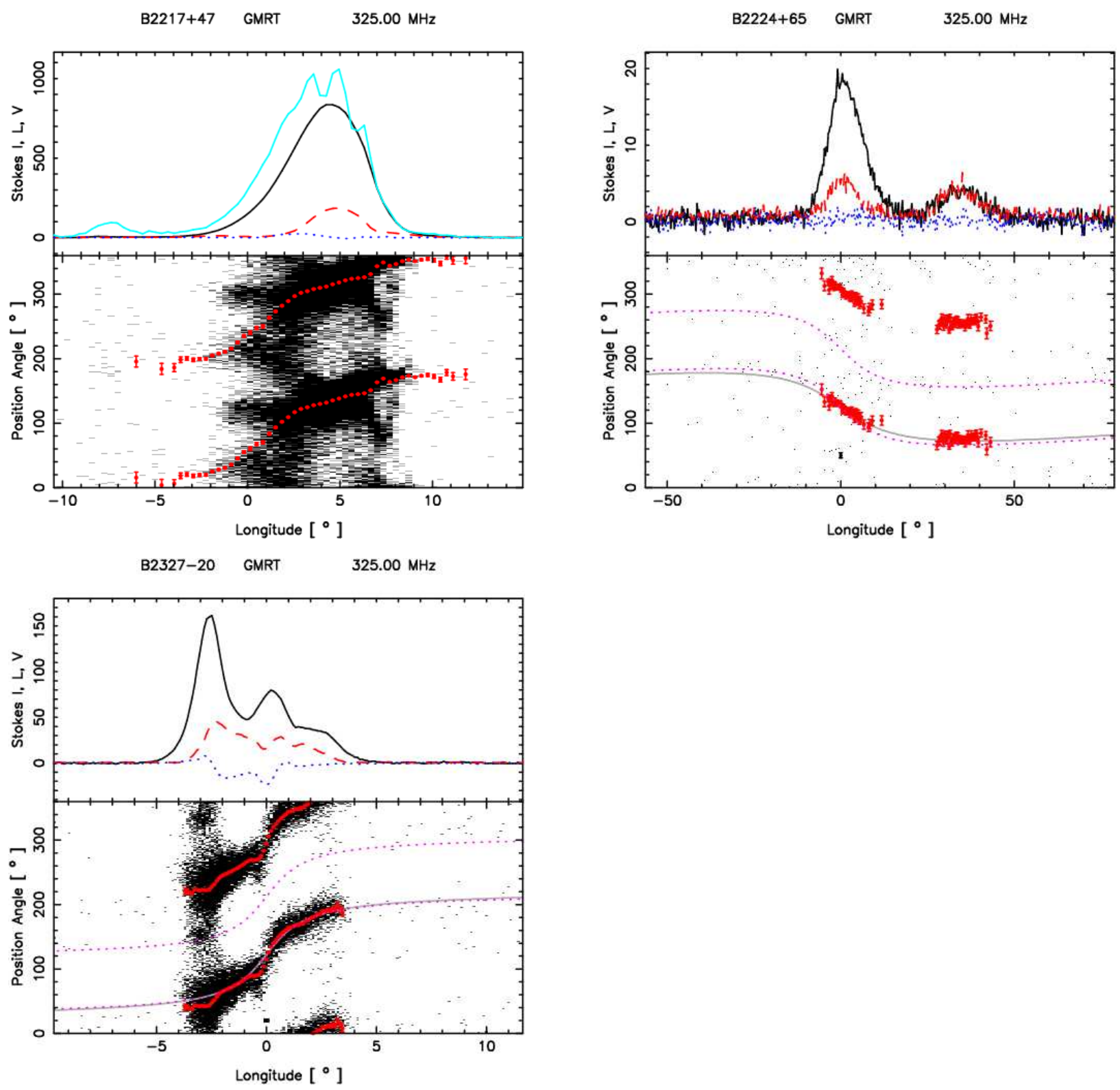

Fig. A10.- PPA histograms and "flared"-emission profiles as in Fig. A1 for pulsars B2217+47, B2224+65 and B2327-20. 
ent point by about $15^{\circ}$, and hence the core peak lags the midway point by about $3^{\circ}$. The overall geometrical evidence here can be understood by invoking effects of $\mathrm{A} / \mathrm{R}$ (BCW as corrected by Dyks et al 2004). If we assume that the flared pulse profile illuminates the full polar cap, then the BCW model gives an emission height for the outer cone of around $494 \mathrm{~km}$. Assuming that the central feature is of the core type, then its peak leads the SG point of the PPA traverse by $12^{\circ}$ yielding a core emission height of about $390 \mathrm{~km}$. These 325-MHz height estimates of a few hundred $\mathrm{km}$ 's are quite reasonable when compared to the radio emission heights estimated in other pulsars.

PSR B0450+55: We have generally viewed this pulsar as having a triple ( $\mathbf{T})$ profile, and its bright component as a core feature marked by sensechanging circular polarization especially at high frequency (e.g., vH, GL, MIS, LM, KL, K-98). However, L\&M were correct to note its forwardshifted PPA traverse at meter wavelengths, such that $\mathrm{A} / \mathrm{R}$ seems to displace its core well toward the leading edge of its profile. The RVM-fitted PPA traverse (see Table A5) gives the SG point at the longitude origin in Figure A1 with an error of about $1^{\circ}$. The about $8^{\circ}$ width of the core at near $1 \mathrm{GHz}$ also constrains $\alpha$ to some $30^{\circ}$. Therefore, here we designate the pulsar as having an arT profile.

Fig. A1 shows the pulsar's profile at $325 \mathrm{MHz}$, where the green curve shows the "flaring" character of the emission on the far trailing edge of the profile, something also seen via the large modulation index in the WES/WSE analysis. The regions immediately adjacent to the bright feature show strong stationery 9.5- $P_{1}$ modulation, suggesting that they are conal. The flared profile gives clear indication of the leading and trailing conal emission, and we can use the edges of the outermost cone to estimate the midway point of the profile which leads the SG point by about $3.9^{\circ}$, in turn giving an emission height of about $275 \mathrm{~km}$. This reasonable height estimate for the conal emission supports the conclusion that $\mathrm{A} / \mathrm{R}$ plays a strong role in the profile evolution.

PSR B0540+23, with its steeply rising profile, long trailing "tail" and flat to steep PPA traverse, is one of LM's classic "partial cone" objects. Moreover, this behavior is progressive over a very broad band from some 0.3 to $10 \mathrm{GHz}$ (RSW, GL, W-99,
W-04, vHX, TR, X93, J-07, BCW), such that at very high frequency the profile has a nearly Gaussian form and an ever more extended "tail" at longer wavelengths. Further, the star's PPA traverse is consistently flatish on the leading side of the profile and rotates ever more steeply downward in the trailing region-perhaps an indicator of $\mathrm{A} / \mathrm{R}$, but also perhaps simply the usual steep PPA rotation under the leading portion of a conal double profile.

Careful inspection reveals that the PPA SG point lags the profile peak ever farther at lower frequencies; for example, at $10.5 \mathrm{GHz}$ the $\mathrm{SG}$ point falls under the symmetrical profile, whereas at 327 $\mathrm{MHz}$ the peak leads the SG point by more than $20^{\circ}$ ! In some low frequency profiles, the trailing "tail" does suggest an unresolved second component (e.g., see GL's profiles at 408 and $234 \mathrm{MHz}$ ); however, our $327-\mathrm{MHz}$ observation in Figure A1 shows little hint of this feature, so this behavior may not be consistent. Overall, we find few signs of conal emission in the star's profiles: no "outriders" are seen at high frequency, no periodic features are seen in its fluctuation spectra (WES/WSE), and the "flaring" on the extreme profile edges is weak.

Fortunately, B0540+23's PSs have been studied carefully at $430 \mathrm{MHz}$ by Nowakowski (1991). Using several different analyses including intensityfractionated profiles, he finds evidence for two or three other regions of emission following the bright component. In Paper VI this pulsar was classified as having a core-single $\left(\mathbf{S}_{t}\right)$ profile; now we tentatively classify it as triple $(\mathbf{T})$. The PPA traverse in Fig. A1 is well fitted by giving an $R$ value of $-3.4^{\circ} \%^{\circ}$ (see Table A5)

Estimating the outside half-power width of the star's full $430-\mathrm{MHz}$ profile as $38^{\circ}$ (see Nowakowski's fig. 4) and scaling to $1 \mathrm{GHz}$ using BCW's profile dimensions measured from the fitted SG points, one obtains the $29^{\circ}$ width value used in Table A3 While a satisfactory solution of the emission geometry can be obtained from this width estimate and fitted $R$ value in the above table, it is interesting to note that the SG point appears to fall well after the profile center. Specifically referring to the $327-\mathrm{MHz}$ profile in Fig. A1, we might expect the scaled half-power width of the putative triple profile to be some $40^{\circ}$, such that the SG point would lag the center by $9^{\circ}$ — very likely suggesting 
that $\mathrm{A} / \mathrm{R}$ is operative in this pulsar's emission.

B0643+80: Profiles for this pulsar have been published spanning $100 \mathrm{MHz}$ to $5 \mathrm{GHz}$ (GL, vH, MIS, MM, S95). Most of these show a bright leading and weak trailing component; however, at 102 $\mathrm{MHz}$ this configuration is reversed, perhaps due to a second mode (MIS). The profile exhibits a nearly constant half power width of about $9^{\circ}$ over this large frequency range, and the PPA sweep rate can be estimated from GL's 1.4-GHz profile. Overall, the pulsar appears to show a conal single evolution that is quite reminiscent of B0943+10. WSE report that the star's fluctuation spectra are featureless at $92 \mathrm{~cm}$. It is thus unsurprising that L\&M classed this pulsar as having a "partial cone" profile.

PSR B0740-28 is yet another example of a "partial cone" in the L\&M sense, apparently owning to the displacement of its PPA SG point on the trailing side of its profile - probably indicative, we now know, of $\mathrm{A} / \mathrm{R}$ effects. Its average profiles show significant pulse-shape evolution with frequency (e.g., $\mathrm{vH}, \mathrm{MHQ}, \mathrm{GL}, \mathrm{J}-05, \mathrm{KJ}, \mathrm{JKW}) \mathrm{A} 1$ and as many as seven components are needed to fit its profile at $1.4 \mathrm{GHz}$ (Kramer 1994).

In Figure $\mathrm{A} 2$ we show the average Stokes profile and grey-scale PPA histogram of the $325-\mathrm{MHz}$ pulse from the GMRT PS. Using a similar analysis as for B0355+54, we failed to find any evidence for "flaring" on the profile edges. Unfortunately, this high S/N profile does resolve the PPA traverse any farther into the "wings" than the previous observations.

More clarifying are the LRF spectra for this pulsar (Fig. 22), computed from the above PS, that exhibit a narrow $3.6 \mathrm{c} / P_{1}$ fluctuation feature on the edges of its profile - suggesting that the pulsar illuminates the entire annular region around its magnetic axis and that its profile edges correspond to the outer edges of this region. No such feature was reported by WES/WSE; but it is possible that such "drifting" intervals are episodic (as for pulsar B1944+17 below), and this may account for this star's profile instability as well.

The RVM fitted PPA gives a maximum sweep rate of $-5.5^{\circ}{ }^{\circ}$ with the resultant SG point at the

\footnotetext{
${ }^{A 1}$ Some of the older published profiles (i.e., MHMA, MHM, MHMb, MGSBT, vO97, even GL) show little detail and thus do not seem to have been resolved adequately.
}

indicated longitude origin (see Table A5). The star's several components (i.e., see KJ's profiles at 1.4 and $3.1 \mathrm{GHz}$ ) can only be understood quantitatively as a core/double structure if its magnetic geometry is nearly orthogonal, such that the inner and outer conal components have outside dimensions of something like 9 and $18^{\circ}$, respectively. We then designate this pulsar as having an arM profile.

B0809+74 presented the defining example of how profile "absorption" (Bartel et al 1981, Bartel 1981) could result in "partial cone" emission. A review of the many consequences as well as modern efforts to understand the effects appears in RRS/RRvLS, which show that the longitude of the magnetic axis at meter wavelengths falls on the leading edge of the profile at about the half-power point; whereas at both higher and lower frequencies the star's profiles appear to be complete. Our GMRT profile in Figure A2 shows the "absorbed" $325-\mathrm{MHz}$ form, such that the full profile would have a half-power width of some $17^{\circ}$. Here, the nearly linear and very highly correlated PPA fit (see Table A5) does not properly locate the fiducial longitude - which here would fall about $-7^{\circ}$ and the PPA "jump" is modal in origin. Despite its "absorption" the star's profile and frequency evolution is characteristic of the conal single $\left(\mathbf{S}_{d}\right)$ class, and $\mathrm{A} / \mathrm{R}$ effects seem to play no significant role. We retain the model of RRS/RRvLS in Table A3.

B0906-17: As seen in Figure A2 the pulsar exhibits a sharply rising profile with a long weak trailing tail as well as a PPA traverse that steepens to the SG point only on the trailing edge of the profile, prompting L\&M to regard it as having "partial cone" emission. We also see evidence of sporadic emission across the entire profile. More recent work (XRSS, vH, GL) often resolves a trailing component, and the $21-\mathrm{cm}$ profiles (see J-05) show a leading-edge inflection that suggests a third. The asymmetrically curved PPA traverse (with a prominent $90^{\circ}$ "jump") steepens steadily with longitude and strongly suggests A/R. Unfortunately, the WES/WSE analyses are not very revealing in this case. The overall evidence then suggests that the star's profile might be regarded as an A/R triple (arT), such that this structure is obscured at low frequency, perhaps because $A / R$ moves some central (putative core- 
component) emission to ever earlier longitudes. The PPA fit (see Table A5) in Fig. A2 is neither able to measure the maximum sweep rate nor to locate the PPA inflection point. That the sweep rate here is far too shallow is clear by reference to the 1.4-GHz profile of J-05.

B0919+06: Figure A2 L\&M classify this pulsar as a "partial cone" with its SG point lying towards the trailing part of its profile. The star's average emission shows a long dim ramp proceeding its bright trailing component, and it exhibits very similar profiles in form and dimensions over the 0.1 to $10.6-\mathrm{GHz}$ range of the existing observations. A recent single pulse polarimetric study (Rankin et al 2006a) found that the dimmer leading parts of the profile can suddenly brighten up for several tens of pulses and then revert back to their normal faintness (see their Figs. 1 and 3). The effect is similar to the "flaring" event seen for PSR B0355+54 discussed earlier, and here the above study demonstrated that the overall profile is triple $(\mathrm{T})$ with both core and conal dimensions scaling in terms of the polar-cap size. The fluctuation spectra provide (see WES/WSE) little insight for this star.

The 325-MHz PS in Rankin et al (2006): fig. 3 clearly shows that the PPAs exhibit strong OPMs mostly towards the leading parts of the profile. The average PPAs thus show a complicated behavior which probably led LM88 to conclude that the SG point is towards the leading edge of the profile. Here, we have used the same $1400-\mathrm{MHz}$ PS as in the above study to fit the RVM to the PPA traverse. The PPA at this frequency is mostly dominated by a single OPM. Our RVM-fitted PPA yields a maximum sweep rate of $+11.8^{\circ} /{ }^{\circ}$ (see Table A5) as shown in Fig. A2 with the longitude origin falling at the SG point towards the trailing edge of the profile. The above sweep rate is steeper and more linear than that seen at meter wavelengths; therefore, we have retained the model values from the above study in Table A3 (see also BCW's profiles). Clearly the star's PPA behavior is consistent with an $\mathrm{A} / \mathrm{R}$ signature as predicted by the $\mathrm{BCW}$ model - so we designate it as having an arT profile - although its overall effect is not at all clear.

B1055-52I: This prominent southern interpulsar has been studied by many investigators (HMAK, MHMA, vO97, Biggs 1990, MHMb, LM, CMH) and the configurations of its main pulse and interpulse widely debated. An interesting comprehensive treatment has been given in the recent paper by Weltevrede \& Wright (2009). These authors find a nearly orthogonal geometry $\left(\alpha=75^{\circ}\right)$ as have several other groups including ourselves (Paper VI). They also support the idea that a trailing portion of the star's interpulse is missing, as did L\&M in arguing that it was a "partial cone". We do not see any flaring towards the leading or trailing edge of the pulsar's interpulse, and also did not manage to get reliable RVM fits to the PPA. However, given the profound differences between the star's main pulse and interpulse propertiestogether with their large widths - we suspect that a solution with a small value of $\alpha$ will ultimately be fully demonstrated. An illustrative such outer cone model for the interpulse is given in in Table A3.

B1112+50: At meter wavelengths this pulsar has an asymmetric single profile, and it is apparently on this basis that L\&M regarded it as a "partial cone". Above $1 \mathrm{GHz}$ the star's profile consists of two components which are at times well resolved and sometimes not, indicating several modes. Profiles and polarimetry are available by a number of authors (MGSBT, GL, L90, XRSS, KL, MIS), and both modal and fluctuation studies are available by Wright et al (1986) and WES/WSE. Our 325-MHz GMRT observation is shown in Figure A3, which shows both its asymmetric single profile and "flared" double form. The LRFs show only weak periodic modulation, but the star's PS are highly modulated at both frequencies in the WES/WSE analyses. Overall, the profile evolution appears conal, though the forms may entail some core emission in the profile center at lower frequencies. In any case, the PPA fitting in Fig. A3 yields a sweep rate of $10^{\circ} /^{\circ}$ and a poorly determined SG point (see Table A5). This together with the profile width and an estimate of the putative core dimension suggest the inner cone geometry in Table A3.

B1221-63: Here, we do not understand why L\&M regarded this pulsar as a "partial cone". Profile polarimetry of uneven quality is available over a band from 0.27 to $1.6 \mathrm{GHz}$ (MHM, MHMA, MHMb, vO97, WMLQ). Overall, the pulsar seems to exhibit a triple form (MHM), and estimates of the profile and core widths together with the sweep 
rate suggest an inner cone geometry as seen in Table A3.

B1240-64: This pulsar has a symmetrical single profile below $1 \mathrm{GHz}$, though some of the observations are poorly resolved (MHMA, CMH, vO97). It was probably the leading "ramp" on MHM's 1.6 $\mathrm{GHz}$ observation that pushed this star into L\&M's "partial cone" category. Surely, KJ's recent 1.4 and $3.1-\mathrm{GHz}$ profiles are the best quality available, and these show perhaps a central notched core flanked, by a leading conal outrider, and just a hint of the trailing one. Further, the PPA traverse above $1 \mathrm{GHz}$ exhibits a perplexing rotation through more than $180^{\circ}$. Despite these difficulties, the star exhibits what is essentially a core-single profile evolution, and the rough $8.4-\mathrm{GHz}$ detection of JKW may show the surviving pair of conal components. If the vO97 profile provides a reliable sweep rate, then the profile dimensions can be roughly squared with an inner cone geometry as shown in the table.

B1322+83m: Little can be gleaned about this star's emission from the published profiles (GL, $\mathrm{KL}$ ); however, the high quality GMRT 325-MHz profile in Figure A3 is more scrutable. The star has two regions of emission, one in the form of a highly polarized "precursor" with a completely flat PPA traverse, and then a second region of emission which is also highly linearly polarized but with a positive sweep rate. We take the position that the precursor is unrelated to the polar-cap core/cone emission structures. Then, the "main pulse" is very likely a conal single profile. This configuration would then be very similar to what is observed in the B0943+10 'Q' mode (Backus et al 2010). The RVM fit (see Table A5) yields a somewhat poorly determined SG point, and its location within the main pulse is consistent with its being close to the profile center.

B1356-60: Some published profiles are useless for our purposes because of scattering or poor resolution (vO97, WMLQ, MHQ). However, the two recent polarized profiles (KJ, JKW) suggest a core-single evolution without conal outriders. Interestingly, KJ find a significant, apparently A/R shift, between 1.4 and $3.1 \mathrm{GHz}$ when the profiles are aligned using their $\mathrm{SG}$ points.

B1426-66: Many published observations are available for this southern pulsar (HMAK, MHMA, MHM, MHMb, vO97, J-07, JKW), and most are of good quality. Apart from its odd profile shape, we cannot see why L\&M saw this star as a "partial cone". Again, it is the Johnston et al (J-05) work that is most insightful. The bright narrow feature marked by antisymmetric $V$ is clearly a core component, and it is flanked by a broad leading component and a weak trailing one. Using the core width to determine $\alpha$, the conal dimensions and the PPA sweep rate, it is clear that an inner cone geometry obtains.

B1449-64: An identical set of observations is available for this prominent southern pulsar (HMAK, MHMA, MHM, MHMb, vO97, J-05, JKW), and while it seems likely that this 180msec pulsar would generate a core feature, no clear circularly polarized signature is apparent. We do see evidence of conal outriders in both the 1.6 (MHM) and 1.4-GHz (J-05) profiles, and the width of the central (putative) core constrains $\alpha$ to some $43^{\circ}$. A rough estimate of the conal outrider dimension then strongly suggests an inner conal geometry.

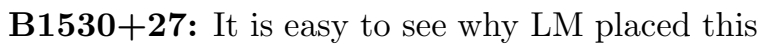
pulsar in their "partial cone" category with its bright leading component and weaker, barely resolved trailing one (RSW, BCW, GL, W-99, W04, MM) - not to mention its weak postcursor. As we see Figure A3, neither its profile nor shallow PPA traverse readily indicate that this could be a conal single or double $(\mathbf{D})$ profile. However, the PRAO profiles (MIS, K-98, KL) show that the trailing component becomes as strong as the first at $100 \mathrm{MHz}$, and HR's time-aligned profiles show how this comes about (properly aligned with a little smaller DM). Moreover, both D86 and WES exhibit the star's prominent correlated subpulse motion, showing that the profile is basically conal.

None of this, though, accounts for the star's weak, highly linearly polarized "postcursor" component, which trails its main emission components by some $50^{\circ}$. Please also note that $\mathrm{W}-04$ shows that the PPA traverse under this feature is nearly constant. We could fit the RVM (see Table A5) to the main pulse however the postcursor emission could not be fitted with the same RVM. The "flaring" analysis did not yield any significant sporadic emission at the profile edges. The SG point appears to be coincident with the profile center as measured with respect to the $10 \%$ outer widths.

B1530-53 has received no recent study, but in- 
deed it appears to present another good example of the "partial cone" emission envisioned by LM. Just as in the case of B0906-17 above, it shows a bright leading and faint trailing component over a broad frequency band (HMAK, MHMA, MHM, $\mathrm{MHMb}, \mathrm{vO} 97)$, and we see also some evidence for both a " $90^{\circ}$ jump" on the leading edge and a steep rotation of the PPA across the middle of the profile. The lowest frequency profiles (MHMb) suggest that the trailing component may increase in relative strength at low frequency. Overall, this pulsar's profile seems to represent a very asymmetric conal double $(\mathbf{D})$ profile.

B1540-06 exhibits an asymmetric single profile with a steep rise and slow fall-off over a very broad frequency range (MHMb, MGSBT, MIS, GL, KL) as also seen in Figure A4. Its linear polarization is small, especially in the trailing part of the profile, and its PPA behavior disorderly and inconsistent-both frequent properties of conal single $\left(\mathbf{S}_{d}\right)$ profiles. It is the WES/WSE work, however, which provides evidence in the form of narrow 0.32 -c $/ P_{1}$ modulation features at both 92 and $21 \mathrm{cms}$. GL's high frequency profiles suggest a sweep rate of about $-14^{\circ} \%^{\circ}$, and the constancy of its profile width over at least four octaves suggests an inner cone geometry.

B1556-44: This is a well studied southern pulsar, and the profile has an asymmetric triple form around $21 \mathrm{cms}$. with a broad central component and weak conal outriders (WMLQ and MHQ) probably prompting L\&M to see it as a "partial cone". At meter wavelengths its profile has a symmetric single form (LM, MHMA, vO97), and at higher frequencies the central (putative core) component is seen to be composed of two overlapping components (MHQ, J-07). The high frequency PPA traverse shows an orthogonal jump below the conal components. Nonetheless, using L\&M's $R$ value and the core width to constrain $\alpha$, we find that an inner cone $\mathbf{S}_{t} / \mathbf{T}$ geometry fits very well. We did not find any "flaring" in the PS.

L\&M considered the star as an example where the SG point is located towards leading side of the profile, and all the above profiles below $1 \mathrm{GHz}$ show this PPA curvature strongly. Interestingly, the PPA histogram observed with the GMRT at $325 \mathrm{MHz}$, shown in Figure A4, does not. The PPA traverse for the central and trailing components is very similar to that at higher frequencies, but shows a non-orthogonal jump (by about $50^{\circ}$ ) below the leading component. This could result from OPM averaging, and the single pulses are not strong enough to distinguish the modes for the conal components. As a consequence no reasonable RVM fit to the PPA swing was possible. However, based on the average PPA traverse, one can readily see the downward trend from the leading to the trailing edge of the profile.

B1604-00 has been studied extensively and can be observed down to $50 \mathrm{MHz}$ and up to at least 5 GHz (MGSBT, MHMA, MHMb, RSW, GL, HW, vO97, vH, MIS, W-99, KL, K-98, MM). L\&M apparently regarded this pulsar has having a "partial cone" profile because of the asymmetric slow rise and steep falloff of its higher frequency profilese.g., see Figure A4. We have earlier regarded this pulsar has being a triple $(\mathbf{T})$, but its profile does not evolve in the usual manner ( $c f$. ., HR), and there is no strong indication to the effect that the middle feature is a core component (R88). Its profile evolution is more suggestive of the conal triple class, as the central component's strength diminishes at low frequency and never dominates the profile. Moreover, while the star's PSs exhibit no clear drift, its subpulses seem to show a kind of "moding" and a long period fluctuation feature (WES/WSE). Overall, we can now best regard B1604-00 as having an inner-conal triple (cT) profile, such that our sightline at meter wavelengths cuts close to the boundary between its two polarization-modal subcones. It is then likely that the weak leading-edge emission is associated with the outer cone as seen in other pulsars with similar geometries [e.g., B0834+06 and B1919+21 (ET VII)]. As in Paper VI, the values in Table $\mathrm{A3}$ are taken from the mode-separated profiles in R88.

$\mathbf{B 1 6 1 2 + 0 7}$ has been observed over a broad band from 0.1 to $5 \mathrm{GHz}$, and overall it exhibits a barely resolved two-component profile with the leading component consistently brighter; see Figure A4 as well as GL, vH, W-99, W-04, MIS, KL and MM. It was this consistent asymmetry that probably caused L\&M to regard it as a "partial cone". Evidence for subpulse drift comes from D86 and WES/WSE. Moreover, the tendency for the star's low frequency profile to have better resolved components further suggests a conal single $\left(\mathbf{S}_{d}\right)$ evolution.

B1641-45: This bright, distant, southern pulsar 
has been observed repeatedly, but at frequencies below $1 \mathrm{GHz}$ its profiles are corrupted by scattering (MHMA, MHM, MHMb, vO97). Only in the 1.4/3.1-GHz profiles of KJ do we begin to see some profile structure, but the conal outriders are far from clear, and the PPA traverses are impossible to decipher. However, the 8.4-GHz polarized profile recently measured by JKW clarifies matters completely. Here we see that the PPA traverse is essentially central, and the outside dimensions of the outrider pair can be reliably determined. This is the basis of the inner-cone $\mathbf{S}_{t}$ geometry determination in Table A3.

B1648-42: Only two observations (vO97, WMLQ) are available for this wide profiled pulsar, and both show two components with a prominently steepening PPA traverse - indeed, probably it was on this basis that L\&M came to regard the pulsar as a "partial cone". Here, we have no basis to decide on whether a trailing portion of the profile is "missing", or whether the profile is complete as it is. In either case a simple geometric model can be assembled to suit the situation: in the first case, probably a c $\mathbf{T}$ would be invoked, and in the latter situation a conal double (D) configuration. Table A3 gives values for the latter case.

B1700-18: In addition to our Figure A5, profiles have been published for this somewhat weak pulsar only by GL, S95 and MM. The star's asymmetrical single profile undoubtedly accounts for its "partial cone" status in L\&M's effort. The strongest evidence, however, comes from WSE, who find drift-associated modulation with a $P_{3}$ of about $3.5 P_{1}$ as well as a strong low frequency modulation feature. The star's profile must then be of the conal single type, and indeed, many such profiles are quite asymmetric. We find some "flaring" in the star's PSs as seen in Fig. A5, and either this pattern or the average profiles can be used to obtain a half-power width of about $12^{\circ}$. Similarly, an RVM fit to the PPA traverse yields a maximum sweep rate of $-8.4^{\circ} \%^{\circ}$ (see Table A5). The SG point lags the center of the outer conal "flared" profile by about $1.7^{\circ}$ - apparently due to $\mathrm{A} / \mathrm{R}$ giving a very reasonable radio-emission height of $279 \mathrm{~km}$.

B1732-07: Figure A5 gives our 325-MHz GMRT profile, and other published observations are available from GL, vH, J-07, and S95. At meter wavelengths the star's profile is somewhat asymmetric, and perhaps this is why L\&M regarded it as a possible "partial cone". In fact, there can now be little doubt but that this star has a triple (T) profile with a central core component. WES/WSE find no evidence of conal modulation features, the star's PPA traverse is highly central, and $\alpha$ can be estimated from the core width. Significant "flaring" can be seen in Fig. A5 that appears to coincide with the three profile components. All these circumstances square in the outer conal geometry of Table A3. The midway point of the "flared" profile leads the peak of the central core component by $2.3^{\circ}$. This gives an $\mathrm{A} / \mathrm{R}$ conal emission height of $205 \mathrm{~km}$ with respect to the core (see G\&G).

B1742-30: This pulsar's geometry has long presented something of a mystery - and indeed it appears to have been to L\&M who listed this star as a possible "partial cone". Several of the older published profiles (MHMA, vO97, XRSS) do not show its full extent, but the long, weak trailing portion is visible in all of LM's observations, that of WMLQ, and the GMRT $325-\mathrm{MHz}$ polarimetry of Figure A5. Nor is it easy to interpret the PPA rotation across the various profiles, but apart from several " $90^{\circ}$ jumps" and the "hat" above the bright, central component, one can interpret the traverse as basically flat and central. The trailing part of the merged main feature thus appears to be a core, with two components preceding it and the two trailing components merged in the long "tail" - reminiscent of B1237+25 in its "abnormal" mode. Fig. A5 further shows most of the five components in the "flaring" analysis, and here the core appears independently enough to measure its half-power width. With all this information and interpretation, the double cone/core geometry of the pulsar is assembled quantitatively in Table A3. Our RVM fits to the PPA does not constrain the SG point at all, as the PPA traverse was essentially a linear slope. We assume that the SG point is close to the peak of the central core component, and hence can quote and error for the PPA offset (see Table A5). An A/R height can be estimated for B1742-30's outer cone with respect to its central core feature (see G\&G). The outer cone's midway point the core peak by some $1.14^{\circ}$, yielding an emission height of $87 \mathrm{~km}$.

B1745-12: The pulsar has been observed at several frequencies by GL, XRSS, S95 and MM. The 
pulsar's highly asymmetric profile at meter wavelengths surely led L\&M to see it as a possible "partial cone"; however, three (or possibly four) components can be discerned at higher frequencies. The GMRT observation at $325 \mathrm{MHz}$ in Figure A5 clearly shows two components as well as a long weak trailing "tail". The PS polarization is weak, but the average shows that the PPA traverse is flat under the leading components (with an OPM "jump" ) but steepens to an SG point near the middle of the overall profile center. We have fitted the OPM-corrected average PPA traverse to the RVM (see Table A5), and the SG point is the longitude origin in the figure.

A search for "flaring" in the trailing portion of the profile identified 14 occasions as indicated by the green curve in Fig. A5, No evidence for periodic modulation was found in the PSs, and indeed the WES/WSE fluctuation spectra are completely featureless. We cannot then be sure about this star's classification, but the weak spreading of its outer conal components appear to reflect an outer cone, and this dimension together with the resultant $-11^{\circ}{ }^{\circ}$ sweep rate provide a basic quantitative geometry in Table A3 which seems compatible with the available observational evidence. Also, the SG point lags the midway point between the outer conal component pair of the "flared" profile by $2.6^{\circ}$ giving an $\mathrm{A} / \mathrm{R}$ emission height for the outer cone of $215 \mathrm{~km}$.

B1756-22: Apart from GL's five polarimetric profiles, only the $1.4-\mathrm{GHz}$ total power observation of S95 has been published; and both spectra of WES/WSE are entirely featureless. The PPA traverse does seem to be nearly flat, and the width of the bright putative core component roughly compatible with an orthogonal magnetic colatitude. Putting this interpretation into Table A3, we find that it is compatible quantitatively with an inner cone/core $\left(\mathbf{S}_{t}\right)$ emission geometry.

B1822-09: With both its interpulse and precursor component, (see Figure A6), this pulsar's geometry has been debated actively since near the time of its discovery. A great many published studies are available (MHMA, MHM, SVS, vO97, MGSBT, MHMb, GL, vH, MIS, KL, K-98, X95, KJ, J-07, WES/WSE), and surely L\&M had adequate reason by virtue of its apparent mainpulse asymmetry to view it as a "partial cone". However, we take the position that the precursor and main pulse are separate entities (Backus et al 2010), so our geometric analysis here follows that in this study and applies only to the main pulse. Our PS analyses of it indicate that this structure represents an inner cone/core triple (T) profile with a nearly central sightline trajectory. An RVM fit to the PPA appeared to be rather complicated for this pulsar, however based on Backus et al's (2010) fig. 8, we argue that the SG point under the main pulse is consistent with being close to the profile center.

B1842+14: Figure A6 gives our 327-MHz Arecibo profile, and many other published observations are available (RSW, vH, GL, HR, MIS, W-99, J-07, MM, WES/WSE). Again, it is not clear what caused L\&M to regard this star as a possible "partial cone", but its flat PPA traverse and steep upturn (see W-99 and J-07) might well have suggested that a further trailing component was missing. Perhaps. The core-single evolution and quantitative geometry fits the pulsar rather well (ET VI) - that is, apart from the unusually flat initial PPA, too sharp upturn, and disparity between the two putative conal components above $1 \mathrm{GHz}$. The delayed upturn might be caused by an $\mathrm{A} / \mathrm{R}$ shift, but this idea does not seem to fit. One other possibility is that the flat PPA represents emission from a highly polarized precursor, and that the remaining parts of the profile represent a core-single structure. However, without quality higher frequency profiles to draw on, this possibility cannot be evaluated. Therefore, we retain the first model in the table.

B1851-14: For this pulsar we have little to go on apart from the few published profiles (L90, XRSS, GL, WSE) and our GMRT 325-MHz profile in Figure A6. Our "educated" guess is that the profile is of the conal single $\left(\mathbf{D}_{d}\right)$ type, and the quantitative geometry in the table is compatible but not well constrained. Some confirmation might come from the fluctuation spectra,but both our own and that of WSE are featureless.

$(\mathbf{B 1 8 5 9 + 0 7 ) :}$ This pulsar was not among the L\&M "partial cone" grouping, but probably would have been included had they known of it. Its asymmetric profile is subject to occasional "events" during which emission in single pulses moves to earlier longitude (see RRW). Otherwise, we found few published references to this pulsar (GL, W-04, WSE), which unfortunately provide 
little further insight. The quantitative geometry in Table A3 is taken verbatim from RRW.

$\boldsymbol{B 1 9 0 0 + 0 5 :}$ Again, this pulsar's asymmetric profiles (MGSBT, RSW, GL, W-99, WES/WSE), also Figure A6, probably encouraged L\&M to regard it as a possible "partial cone". Beyond this, there is little clear evidence to go on. Both Paper VI and W-99 classified it as a core-single star, but no core signature can be discerned in any of the existing profiles. This classification still seems likely, but the profile width - and thus the geometrical model - in Paper VI are incorrect. We have repaired this error above in Table A3 on the basis of revised estimates.

B1907-03: Here the published studies clearly show a core/cone triple $\left(\mathbf{S}_{t}\right)$ profile at $21 \mathrm{cms}$. (GL, S95), whose core is even marked by signchanging circular polarization, and a single profile at $408 \mathrm{MHz}$ (L90). Our GMRT 325-MHz profile in Figure A7, unfortunately, is useless owing to its distortion by scattering, and no useful information comes from the fluctuation spectra in WSE. Most observations suggest a flat, central PPA traverse, and this together with the profile dimensions fixes an inner cone/core geometry quantitatively.

B1910+20: The pulsar was classified as a "partial cone" by L\&M with the SG point lying towards the trailing edge of the profile. Average profiles at 610 and $1410 \mathrm{MHz}$ (GL) show a strong leading and weak trailing component. These PPA traverses appear complex such that no clear interpretation can be made. However, the $1.4-\mathrm{GHz}$ profiles of W-99, RSW and this paper, Figure A7. show the full PPA behavior in some detail. The latter in particular is complex and cannot be described by the smooth RVM curve, but the expected underlying ' $\mathrm{S}$ ' shape is evident — and our efforts to fit the RVM to the PPM traverse yield the SG point with resonable precision (see Table A5).

The available pulse-modulation studies (D86, WES/WSE) leave no doubt that the profile represents a double conal structure: the outer conal components show a fairly regular stationary modulation with a $P_{3}$ of about $2.7 P_{1}$, and we see evidence (e.g., DHCR) that this modulation is shared by the inner conal components as well. We have found no evidence of flared emission at the profile edges. Our LRF spectra in Fig. 2 yields signatures of the 2.7- $P_{1} P_{3}$ modulation in the outer conal component pair.
As no clear signature of a core component can be seen in any profile, its width cannot be determined. We do see hints of core activity including antisymmetric $V$ in some profiles, but overall we cannot resolve whether this profile is of the $\mathbf{M}$ or cQ type. However, using the PPA fit above and the profile dimensions from Paper VI, a slightly revised quantitative model of the emission geometry can be found in Table A3. Further our measurements show that the midway point leads the SG point by only $0.5^{\circ}$, giving an emission height of about $228 \mathrm{~km}$.

B1913+10: Little more can be said about this pulsar's geometry than was possible in Paper VI. The $4.85-\mathrm{GHz}$ profile is so poorly resolved that no structure can be seen, and the $400-\mathrm{MHz}$ profiles have scattering "tails". The recent, well measured profiles of J-07, W-99 and Figure A7 resolve a feature on the profile's trailing edge at $1.4 \mathrm{GHz}$ that becomes very pronounced at $3.1 \mathrm{GHz}$. The one available LRF (WSE) is featureless. It still may be that this is a core-single star, but no geometrical solution bears this out. The two resolved components at $3.1 \mathrm{GHz}$ cannot be interpreted as a conal outrider pair: their outside dimension is much too small for them to be an inner cone.

B1915+13: In slighly poorer observations this pulsar exhibits only a single narrow Gaussianshaped component, but when resolved optimally it has an unresolved feature on its trailing edge. This structure is clearly seen in the $1.4-\mathrm{GHz}$ profiles of BCW, EW, W-99, HR and Figure A7 and these and many other observation also show an accelerating PPA rotation such that the SG point falls far on the trailing edge of the profile (GL, RSW, RB, vH) - and very like that of $\mathrm{B} 0540+23$ above where the shift increases with wavelength. PPA fits by BCW and EW as well as ourselves at $1.4 \mathrm{GHz}$ consistently show that the SG point falls far on the trailing edge of its profile, and GL's lower frequency profiles suggest even greater displacements, such that $\mathrm{A} / \mathrm{R}$ effects provide a natural explanation. The star's fluctuation spectra are featureless (WES/WSE), and the weak "flaring" in the above figure does not seem indicative of conal emission. Interestingly, the pulsar has been detected down to $100 \mathrm{MHz}$ (KL, MIS). For all these reasons we classify this star as having an arSt profile.

B1924+16: This pulsar exhibits a single com- 
ponent with a long slow rise on its leading edge. The published profiles give a mixed impression regarding the curvature of PPA traverse (RB, BCW, RSW, GL, vH, W-99), but the fits by BCW and ourselves in Figure A8 concur in showing a slight upward acceleration and thus placing the SG point toward the trailing edge of the profile (the longitude origin in the above figure). Weak indication of a long (about $60 P_{1}$ modulation) is seen in the fluctuation spectra (WES/WSE), but overall there is little indication of conal activity. On this basis we designate the pulsar as having an arSt profile. The RVM fit to the PPA yields the SG point with resonable accuracy (see Table A5). The midway point of the profile calculated using the outer peaks of the "flared" profile leads the SG point by roughly $4.1^{\circ}$, giving an $\mathrm{A} / \mathrm{R}$ height of about 506 $\mathrm{km}$.

B1930+22: This fast, highly dispersed pulsar is difficult to observe at lower frequencies (although $\mathrm{MM}$ report a $100-\mathrm{MHz}$ detection), and only the 1.4-GHz profiles of GL, BCW, W-99 and the AO observation in Figure A8 fully show its fast rise and slower falloff. Several of the observations suggest an upwardly curved PPA traverse, and our RVM fit places the SG point towards the trailing side of the profile. The significance of this placement is not yet clear: One might attribute this configuration to $\mathrm{A} / \mathrm{R}$, however, the "flaring" on the two edges of the profile suggests some conal activity there. Possibly $\mathrm{A} / \mathrm{R}$ does shift some highaltitude core emission earlier so as to overlie the leading conal feature, but new high quality observations are needed at lower frequencies to assess this possibility. We then retain the $\mathbf{S}_{t}$ designation of Paper VI but amend it to show the probable role of abberation/retardation. Using both the "flared" and the average profiles, the center of the conal peaks lead the SG point by $8.3^{\circ}$. This shift provides an A/R-height estimate of $250 \mathrm{~km}$ for the conal emission.

B1937-26 shows a consistently asymmetric profile that prompted L\&M to regarding it as a "partial cone" (GL, WMLQ, vO97, vH, MHQ). A bright leading and weak trailing feature are seen in all the star's profiles, but in several of the higher frequency profiles (including the J-05 that is best resolved), we see a suggestion of a third feature on the leading edge. Further, the fluctuation spectra (WES/WSE) suggest conal emission as does the "flaring" in our GMRT 325-MHz observation in Figure A8. We cannot then resolve just how the profile should be classified, but using the fitted PPA sweep rate and conjecturing that the high frequency profile width reflects the core width, the geometry in the table is compatible quantitatively with an inner cone. RVM PPA fitting to this shallow PPA traverse does not constrain the SG point well enough (see Table A5). Nonetheless, using the "flared" and average profiles the midway point of the profile leads the SG point by $0.8^{\circ}$ giving an $\mathrm{A} / \mathrm{R}$ height estimate of about $70 \mathrm{~km}$.

B1944+17: The pulsar's main-pulse profile (see Figure A8) superficially resembles some of the conal "partial cone" objects we have identified above (e.g., B1540-06) with weak emission on their trailing sides ( $c f$. ., HR), but the detailed published studies leave little doubt that this star is correctly classed as having a conal triple/quadruple $(\mathrm{c} \mathbf{T} / \mathrm{c} \mathbf{Q} /)$ profile. The pulsar has several modes, some with orderly drift (Deich et al 1986; WES/WSE), and these together with its $\gtrsim 60 \%$ null pulses make its profiles somewhat unstable (MHMA, MHM, MGSBT, RSW, vO97, vH, W-99, MIS). Its shallow, linear PPA rotation and orderly profile evolution (e.g., HR) further support this understanding of its emission geometry. Indeed, in a recent study by Kloumann \& Rankin (2010) the pulsar's geometry has been analyzed in detail; the values in Table A3 are taken from this work. Our RVM fittig results are given in Table A5.

B1944+22: The two existing AO profiles (RB, $\mathrm{W}-99$ ) of this weak pulsar reveal only that it has two unresolved components - much as seen in Figure A9 the second of which is much weaker. The profile is almost certainly conal, and thus its behavior is very likely akin to that of the many conal single (or inner-cone double) stars with weak or missing trailing emission. If the $\mathrm{W}-99 \mathrm{PPA}$ rate is reliable, then we can easily compute a model for the star's geometry as in Table A3.

B2021+51: This bright pulsar has been studied for many years, and most evidence points to its having a conal single $\left(\mathbf{S}_{d}\right)$ profile that shows the characteristic low frequency bifurcation with a much weaker leading component (M71, MGSBT, vH, GL, X-95, KL, MM, K-98); see especially K98. This behavior is thus very similar to that of B0809+74 (e.g., RRS). Both its SVM PPA 
traverse and subpulse-drift modulation (ETIII; WES/WSE) are also largely compatible with this understanding. Interestingly (and unusually) the pulsar's leading edge emission is fully linearly polarized at meter wavelengths (as is B0809+74's at frequencies above $1 \mathrm{GHz}$ ) such that the one active polarization mode here must be completely linearly polarized. Note in Figure A9 that both OPMs are active only under the trailing component. In Table A3 we revise slightly our earlier emission model in Paper VI: surely the pronounced conal spreading seen in K-98 argues for an outer-cone geometry. In Table $\mathrm{A5}$ we give the RVM fitted parameters, and the SG point seems to be well constrained. The "flaring" analysis for this star shows only weak, occasional emission on the profile edges. These do provide a means of estimating the profile's midpoint - which leads the fitted SG point by about $1.1^{\circ}$ —giving an $\mathrm{A} / \mathrm{R}$ emission-height estimate of $113 \mathrm{~km}$.

B2043-04: The published profiles of this pulsar all show a symmetrical single form with a "soft" leading edge (GL, vO97, MIS). It is thus not very clear why L\&M regarded it as a possible "partial cone". The 325-MHz GMRT profile in Figure A9 is the only one that it well enough resolved to indicate two features as well perhaps as "flaring" on its leading edge. There can be little doubt that the profile is conal, probably an inner cone $\mathbf{S}_{d}$ with occasional outer conal subpulses on the far leading edge (as seen in some other such pulsars, e.g., see B1604-00). This understanding is corroborated strongly by WES/WSE's analyses showing a strong narrow fluctuation feature at $0.37 \mathrm{c} / P_{1}$ that is clearly indicative of subpulse drift. The SG point can be fixed by an RVM PPA fit (see Table A5) as shown in Fig. A9. The midpoint of the outer conal peaks of the "flared" profile coincides with the SG point within the measurement errors, suggesting that $A / R$ is not significant in this slow pulsar.

B2053+36: This pulsar's asymmetric single profile seems to have been the reason for L\&M's "partial cone" categorization (RSW, GL, W-99). And, indeed, it is also problematic from our perspective. Its flat segmented PPA traverse is unusual and apparently indicative of a central sightline traverse, even if the "jump" is due to an OPM dominance transition as indicated in Figure A9, Entirely conal profiles are rare in pulsars with such a short period, but we see no hint of core action. Moreover, WES/WSE make a claim for subpulse drift without direction! However, they find no consistent behavior at their two frequencies. No consistent geometrical model can be computed from the available information.

B2217 +47 exhibits a somewhat asymmetrical profile over a broad band, and this apparently led L\&M to see it as a "partial cone" (GL, SVS, MIS, MM). Our GMRT 325-MHz profile in Figure A10 shows a similar form. We see some hint of conal outriders at $21 \mathrm{~cm}$ (K-98 and MGSBT) and these appear to dominate the profile at 4.9 $\mathrm{GHz}$ (SRW) - arguing strongly that the star is a member of the core-single $\left(\mathbf{S}_{t}\right)$ class. Interestingly, WES/WSE find some evidence for systematic subpulse motion at $1.4 \mathrm{GHz}$, but without a fuller study their result is hard to interpret. These results then provide the needed information to construct the quantitative emission model given in Table A3

$\mathrm{B} 2217+47$, however, shows further unorthodox behaviors that need further study. Downs (1979) found that there was a strange truncatedexponential baseline emission that decayed after the pulse, and MGSBT's profiles were not sensitive enough fully confirm or refute it. Moreover, SS find that the star has a postcursor feature was variable in its intensity and position over a few years. Our search however did not show such a feature in our data.

B2224+65m: This pulsar has two well separated Gaussian-shaped components (MGSBT, GL, LM, vH, K-98, KL, MM) as seen in Figure A10. The trailing one, however, has a flat PPA, apparently causing L\&M to see the profile as a "partial cone" with a missing leading component. Indeed, we classified it as having a $T_{1 / 2}$ profile in Paper VI. Clearly, we must now view the fully linearly polarized trailing component as being a "postcursor" feature, and the much less polarized leading component as a "main pulse" in its own right. The PPA fit (see Table A5) in the figure does seem to fit both features well, but their separation is large some $35^{\circ}$. This said, we can only estimate $\alpha$ from the width of the putative core main pulse. We see no hint of conal outriders, and WES/WSE report featureless fluctuation spectra. We note that $\mathrm{vH}$ finds this pulsar similar to $\mathrm{B} 0355+54$; however, we see no evidence of the $A / R$ which 
is prominent in that star's PPA traverse. The SG point in this pulsar is consistent with being coincident with the profile center of the main pulse.

B2327-20: As shown in Figure A10 the pulsar has a triple profile with a weak trailing component. This is much clearer in the well resolved GMRT profile than in many of the published ones (MHMA, MHMb, MGSBT, vH, vO97, GL, CMH), especially at high frequency where the weak component can be gleaned only as an inflection on the trailing edge. Only J-07's $691-\mathrm{MHz}$ profile provides comparable clarity. Clearly, this star became one of L\&M's best examples of "partial cone" profiles. The remaining question is whether the star has an entirely conal triple profile or a core-cone triple one, and this question is difficult to fully resolve. However, the intensity dependence of the central feature and its aberrant PPA behavior tilts in favor of it being a $\mathbf{T}$ pulsar. WES/WSE find a $50-P_{1}$ feature shared by both the leading and middle components that could be null-related, whereas the weaker $0.39-\mathrm{c} / P_{1}$ modulation seems to be present only in the leading component. Apart from the now much better measured PPA rate, taken from the fit in the figure, the quantitative geometry in Table A3 follows the earlier analysis in Paper VI. Our RVM fit to the PPA traverse yields well determined SG point (see Table A5) and the center of the outer conal peaks coincide with the SG point within the measurement errors. This behavior is consistent with other slow pulsars showing little or no $\mathrm{A} / \mathrm{R}$ effect. 